\title{
Transition Parameters for Doubly Ionized Lutetium
}

\author{
B. KARAÇOBAN* AND L. ÖZDEMÍR \\ Department of Physics, Sakarya University, 54187, Sakarya, Turkey \\ (Received February 24, 2012; in final form February 12, 2013)
}

\begin{abstract}
Using the relativistic Hartree-Fock method developed by Cowan, we calculated the electric dipole (E1) transition parameters such as the wavelengths, the weighted oscillator strengths, and the transition probabilities (or rates) for doubly ionized lutetium ( $\mathrm{Lu}$ III, $Z=71$ ). We compared the results with available calculations and experiments in literature. A discussion of these calculations for Lu III in this study has also been made in view of the relativistic Hartree-Fock method.
\end{abstract}

DOI: 10.12693/APhysPolA.123.651

PACS: 31.15.ag, 31.15.aj, 31.30.-i, 32.70.Cs

\section{Introduction}

There is an increasing need for accurate spectroscopic data on rare-earth elements. Such data are essential in various fields: nucleosynthesis of heavy elements in chemically peculiar stars, stellar structure and evolution, research on light because of rich emission spectra, and diagnostics and design of high-density discharge lamps where rare-earth salts are used. The development and application of reliable methods for theoretical investigation of rare-earth elements is highly desirable. The methods have to consider correlation and relativistic effects simultaneously, because both play an important role in the spectra of heavy elements. Simultaneous treatment of these effects requires many more configurations and high $l$ orbitals. In this case, the expansions from mixing configurations are very complex, therefore, and are limited by computer constraints.

In the sequence of doubly ionized rare earths, lutetium immediately follows the completion of the $4 f$ shell. The levels due to the single outer electron exhibit the properties of this sequence in that their configuration position and spin-orbit interactions are end points for the regular variation of these properties. This simple one-electron spectrum is superimposed on the complex three-electron spectrum arising from the excitation of an electron out of the $4 f$ shell [1].

Doubly ionized lutetium (Lu III) is characterized by a simple atomic structure with a full $4 f^{14}$ subshell appearing in the core and only one outer electron. There is substantial spectroscopic literature concerning Lu III, though less than for the neutral or singly ionized species. The work of Meggers and Scribner on the arc and spark spectra of lutetium revealed, for the first time, lines and levels of Lu III [2, 3]. Steudel proposed interchange of the classifications of two of these lines at 2563.53 and $2603.34 \AA[4]$. The one-electron spectra of doubly ionized lutetium were studied experimentally by Kaufman and Sugar [1, 5]. Later, they determined the $4 f^{14} 5 f-4 f^{14} 5 g$ transitions in Lu III [6]. Migdalek

*corresponding author; e-mail: bkaracoban@sakarya.edu.tr studied influence of core polarisation on relativistic oscillator strengths for the $4 f^{14} 6 s^{2} S_{1 / 2}-4 f^{14} 6 p^{2} P_{1 / 2,3 / 2}^{\circ}$ transitions in Lu III spectrum [7], and reported calculations of relativistic model-potential ionized energies and transition probabilities in the one-electron spectrum of doubly ionized lutetium [8]. Relativistic ionized energies and fine structure intervals of $4 f^{14} n l$ states in Lu III were computed including correlations within the Rayleigh-Schrödinger perturbational approach by Koc and Migdalek [9]. Quinet and Biémont calculated Landé $g$-factors for experimentally determined energy levels in doubly ionized lutetium [10]. The radiative lifetimes of the $4 f^{14} 6 p_{1 / 2}$ and $4 f^{14} 6 p_{3 / 2}$ levels, oscillator strengths and transition probabilities in Lu III were calculated by Biémont et al. [11]. Fedchak et al. measured radiative lifetimes for the $4 f^{14} 6 p_{1 / 2}$ and $4 f^{14} 6 p_{3 / 2}$ levels of Lu III using time-resolved laser-induced fluorescence method [12].

Our aim here is to determine the radiative properties, such as the wavelengths, oscillator strengths, and transition probabilities, for electric dipole transitions (E1) in Lu III $(Z=71)$. These calculations have been performed by using code [13] developed by Cowan for relativistic Hartree-Fock (HFR) calculations [14]. This code considers both correlation effects and relativistic corrections. These effects contribute importantly to the physical and chemical properties of atoms or ions, especially lanthanides. The open-shell configuration structure of Lu III is similar to that of La III except for the $4 f$ shell in the core configuration. Its ground state is $6 s^{2} S_{1 / 2}$, with a closed shell formed by the $14 f$ electrons. We studied two configuration sets according to valence excitations and core-valence correlations for correlation effects. For core-valence correlation, we have only taken into account the configurations including one electron excitation from $4 f$ subshell to other high subshells: $4 f^{14} \mathrm{~ns}$ $(n=6-10), 4 f^{14} n p(n=6-10), 4 f^{14} n d(n=5-10)$, $4 f^{14} n g(n=5-10), 4 f^{14} n f(n=5-10), 4 f^{13} 5 d 6 p$, $4 f^{13} 6 s 6 p, 4 f^{13} 5 d 6 s, 4 f^{13} 6 s^{2}$, and $4 f^{13} 5 d^{2}$. For valence excitations, we have only taken into account the configurations including one electron excitation from valence to other high subshells: $4 f^{14} n s$ ( $\left.n=6-30\right), 4 f^{14} n p$ $(n=6-30), 4 f^{14} n d(n=5-30), 4 f^{14} n g(n=5-30)$, and $4 f^{14} n f(n=5-30)$. These sets are denoted by $\mathrm{A}$ 
for core-valence correlation and B for valence excitations, respectively, in Tables I and II. We presented the energies, Landé $g$-factors, and lifetimes for $4 f^{14} n s$ $(n=6-30), 4 f^{14} n d, 4 f^{14} n g, 4 f^{14} n f(n=5-30)$, and $4 f^{14} n p(n=6-30)$ excited levels of Lu III [15]. In addition, we reported various atomic structure calculations such as energy levels, transition energies, hyperfine structure, lifetimes, and electric dipole transitions for some lanthanides (La I-III, Lu I-III, and Yb I-III) [16-27].

\section{Calculation method}

An electromagnetic transition between two states is characterized by the angular momentum and the parity of the corresponding photon. If the emitted or absorbed photon has angular momentum $k$ and parity $\pi=$ $(-1)^{k}$, then the transition is an electric multipole transition $(\mathrm{E} k)$. However, if the photon has parity $\pi=(-1)^{k+1}$ the transition is a magnetic multipole transition $(\mathrm{M} k)$.

The transition rate (or probability) [28] for emission due to a transition from an upper level to a lower level is given by

$$
\begin{aligned}
& A^{\pi k}\left(\gamma^{\prime} J^{\prime}, \gamma J\right)=2 C_{k}\left[\alpha\left(E_{\gamma^{\prime} J^{\prime}}-E_{\gamma J}\right)\right]^{2 k+1} \\
& \times \frac{S^{\pi k}\left(\gamma^{\prime} J^{\prime}, \gamma J\right)}{g_{J^{\prime}}}
\end{aligned}
$$

and emission oscillator strength [28] is given by

$$
\begin{aligned}
& f^{\pi k}\left(\gamma^{\prime} J^{\prime}, \gamma J\right)=-\frac{1}{\alpha} C_{k}\left[\alpha\left(E_{\gamma J}-E_{\gamma^{\prime} J^{\prime}}\right)\right]^{2 k-1} \\
& \quad \times \frac{S^{\pi k}\left(\gamma^{\prime} J^{\prime}, \gamma J\right)}{g_{J^{\prime}}},
\end{aligned}
$$

where $C_{k}=(2 k+1)(k+1) / k((2 k+1) ! !)^{2}$, and $g_{J^{\prime}}$ denotes the statistical weight of the upper level, namely $g_{J^{\prime}}=$ $2 J^{\prime}+1$. In addition, $\alpha$ is the fine structure constant, and $\gamma$ denotes the configuration (seniority, configuration, coupling scheme, etc.). If each multipole is described by transition operator $O_{q}^{\pi(k)}$ (a spherical operator of rank $k$ and parity $\pi$ ), the line strength [28] can be written as

$$
S^{\pi k}\left(\gamma J, \gamma^{\prime} J^{\prime}\right)=\sum_{M, M^{\prime}, q}\left|\left\langle\gamma J M\left|O_{q}^{\pi(k)}\right| \gamma^{\prime} J^{\prime} M^{\prime}\right\rangle\right|^{2} .
$$

The strongest transition rate (or probability) is electric dipole (E1) radiation. For this reason, the E1 transitions are understood as being "allowed", whereas high-order transitions are understood as being "forbidden".

In HFR method, for an $N$ electron atom of nuclear charge $Z_{0}$, the Hamiltonian is expanded as

$$
H=-\sum_{i} \nabla_{i}^{2}-\sum_{i} \frac{2 Z_{0}}{r_{i}}+\sum_{i>j} \frac{2}{r_{i j}}+\sum_{i} \zeta_{i}\left(r_{i}\right) \boldsymbol{l}_{i} \cdot \boldsymbol{s}_{i}
$$

in atomic units, with $r_{i}$ the distance of the $i$-th electron from the nucleus and $r_{i j}=\left|\boldsymbol{r}_{i}-\boldsymbol{r}_{j}\right| . \zeta_{i}(R)=\frac{\alpha^{2}}{2} \frac{1}{r}\left(\frac{\partial V}{\partial r}\right)$ is the spin-orbit term, with $\alpha$ being the fine structure constant and $V$ the mean potential field due to the nucleus and other electrons.

In this method, one calculates single-configuration radial functions for a spherically symmetrised atom (center-of-gravity energy of the configuration) based on the
Hartree-Fock method. The radial wave functions are also used to obtain the atom's total energy $\left(E_{\mathrm{av}}\right)$ including approximate relativistic and correlation energy corrections. Relativistic terms in the potential function give approximate relativistic corrections to the radial functions, as well as improved relativistic energy corrections in heavy atoms. In addition, a correlation term is included to make the potential function more negative, thereby helping to bind negative ions. These radial functions are also used to calculate Coulomb integrals $F^{k}$ and $G^{k}$ and spinorbit integrals $\zeta_{n l}$. After radial functions have been obtained based on the Hartree-Fock model, the wave function $|\gamma J M\rangle$ of the $M$ sublevel of a level labeled $\gamma J$ is expressed in terms of $L S$ basis states $|\alpha L S J M\rangle$ by the formula

$$
|\gamma J M\rangle=\sum_{\alpha L S}|\alpha L S J M\rangle\langle\alpha L S J \mid \gamma J\rangle .
$$

If determinant wave functions are used for the atom, the total binding energy is given by

$$
E=\sum_{i}\left(E_{k}^{i}+E_{n}^{i}+\sum_{j<i} E^{i j}\right),
$$

where $E_{k}^{i}$ is the kinetic energy, $E_{n}^{i}$ is the electron-nuclear Coulomb energy, and $E^{i j}$ is the Coulomb interaction energy between electrons $i$ and $j$ averaged over all possible magnetic quantum numbers.

In this method, relativistic corrections have been limited to calculations to the mass-velocity and the Darwin corrections by using the relativistic correction to total binding energy. The total binding energy is given by formulae (7.57), (7.58), and (7.59) in [14].

\section{Results and discussion}

We calculated the transition parameters (wavelengths, oscillator strengths, and transition probabilities) for electric dipole (E1) transitions in Lu III $(Z=71)$ using HFR code [13]. Two different calculations have been here performed to obtain configuration state functions (CSFs) according to core-valence correlation and valence excitations. These calculations have been denoted by A and B letters, respectively. We have taken into account $4 f^{14} \mathrm{~ns}$ $(n=6-10), 4 f^{14} n p(n=6-10), 4 f^{14} n d(n=5-10)$, $4 f^{14} n g(n=5-10), 4 f^{14} n f(n=5-10), 4 f^{13} 5 d 6 p$, $4 f^{13} 6 s 6 p, 4 f^{13} 5 d 6 s, 4 f^{13} 6 s^{2}$, and $4 f^{13} 5 d^{2}$ configurations for the calculation $\mathrm{A}$, and $4 f^{14} n s(n=6-30), 4 f^{14} n p$ $(n=6-30), 4 f^{14} n d(n=5-30), 4 f^{14} n g(n=5-30)$, and $4 f^{14} n f$ ( $\left.n=5-30\right)$ configurations for the calculation $\mathrm{B}$ outside the core [Xe] in Lu III. Table I and Table II display transition parameters between low-lying levels. References for other comparison values are indicated below the tables with a lowercase superscript; odd-parity states are indicated by the superscript "o".

Electron correlation effects and relativistic effects play an important role in the spectra of heavy elements. To accurately predict the radiative atomic properties for heavy atoms such as Lu III, complex configuration interactions and relativistic effects must be considered simultaneously. Although Cowan's approach is based on 
Schrödinger's equation, it includes the most important relativistic effects like mass-velocity corrections and the Darwin contributions. Also, for complex atoms, it is important to allow for spin-orbit interaction, which represents the magnetic interaction energy between electron's spin magnetic moment and the magnetic field that the electron sees due to its orbital motion through the electric field of the nucleus. These contributions are considered as perturbations. Thus, to solve the Schrödinger equation with this Hamiltonian, we define a new angular momentum operator in an intermediate coupling scheme.
In calculations, the calculated eigenvalues of Hamiltonian were optimized to the observed energy levels via a least-squares fitting procedure using experimentally determined energy levels, specifically all of the levels from the NIST compilation [29]. The scaling factors of the Slater parameters $\left(F^{k}\right.$ and $\left.G^{k}\right)$ and of configuration interaction integrals $\left(R^{k}\right)$, not optimized in the least-squares fitting, were chosen equal to 0.85 , while the spin-orbit parameters were left at their initial values. This low value of the scaling factors has been suggested by Cowan for neutral heavy elements [14].

TABLE I

Wavelengths, $\lambda$, weighted oscillator strengths, $g f$, and weighted transition probabilities, $g A_{k i}$, for electric dipole (E1) transitions in Lu III.

\begin{tabular}{|c|c|c|c|c|c|c|c|}
\hline \multicolumn{2}{|c|}{ Transition } & \multicolumn{2}{|c|}{$\lambda[\AA]$} & \multicolumn{2}{|c|}{$g f$} & \multicolumn{2}{|c|}{$g A_{k i}\left[\mathrm{~s}^{-1}\right]$} \\
\hline Lower level & Upper level & This work & Others & This w. & Others & This work & Others \\
\hline \multirow[t]{4}{*}{$4 f^{14} 6 s^{2} S_{1 / 2}$} & $4 f^{14} 6 p^{2} P_{1 / 2}^{\circ}$ & $2604.13^{\mathrm{A}, \mathrm{B}}$ & $2603.35^{a}$ & $0.706^{\mathrm{A}}$ & $0.630^{a}$ & $6.94 \times 10^{8 \mathrm{~A}}$ & $6.20 \times 10^{8 a}$ \\
\hline & & & $2603.2725^{b}$ & $0.833^{\mathrm{B}}$ & $0.806^{f}$ & $8.19 \times 10^{8 \mathrm{~B}}$ & $7.82 \times 10^{8 c}$ \\
\hline & & & & & $0.808^{g 1}$ & & $6.02 \times 10^{8 d}$ \\
\hline & & & & & $0.590^{g 2}$ & & $5.10 \times 10^{8 e}$ \\
\hline \multirow[t]{4}{*}{$4 f^{14} 6 s^{2} S_{1 / 2}$} & $4 f^{14} 6 p^{2} P_{3 / 2}^{\circ}$ & $2236.88^{\mathrm{A}}$ & $2236.18^{a}$ & $1.616^{\mathrm{A}}$ & $1.468^{a}$ & $2.15 \times 10^{9 \mathrm{~A}}$ & $1.96 \times 10^{9 a}$ \\
\hline & & $2236.87^{\mathrm{B}}$ & $2236.135^{b}$ & $1.939^{\mathrm{B}}$ & $1.834^{f}$ & $2.59 \times 10^{9 \mathrm{~B}}$ & $2.40 \times 10^{9 c}$ \\
\hline & & & & & $1.846^{g 1}$ & & $1.89 \times 10^{9 d}$ \\
\hline & & & & & $1.388^{g 2}$ & & $1.63 \times 10^{9 e}$ \\
\hline \multirow[t]{3}{*}{$4 f^{14} 5 d^{2} D_{3 / 2}$} & $4 f^{14} 6 p^{2} P_{1 / 2}^{\circ}$ & $3058.76^{\mathrm{A}}$ & $3057.87^{a}$ & $0.465^{\mathrm{A}}$ & $0.388^{a}$ & $3.31 \times 10^{8 \mathrm{~A}}$ & $2.77 \times 10^{8 a}$ \\
\hline & & $3058.75^{\mathrm{B}}$ & $3057.856^{b}$ & $0.516^{\mathrm{B}}$ & & $3.68 \times 10^{8 \mathrm{~B}}$ & $3.76 \times 10^{8 c}$ \\
\hline & & & & & & & $3.10 \times 10^{8 d}$ \\
\hline \multirow[t]{3}{*}{$4 f^{14} 5 d^{2} D_{3 / 2}$} & $4 f^{14} 6 p^{2} P_{3 / 2}^{\circ}$ & $2564.26^{\mathrm{A}}$ & $2563.49^{a}$ & $0.110^{\mathrm{A}}$ & $0.093^{a}$ & $1.11 \times 10^{8 \mathrm{~A}}$ & $\begin{array}{l}2.76 \times 10^{7} \\
9.40 \times 10^{7 a}\end{array}$ \\
\hline & & $2564.25^{\mathrm{B}}$ & $2563.508^{b}$ & $0.123^{\mathrm{B}}$ & & $1.25 \times 10^{8 \mathrm{~B}}$ & $1.11 \times 10^{8 c}$ \\
\hline & & & & & & & $\begin{array}{l}9.68 \times 10^{7 d} \\
8.96 \times 10^{7 e}\end{array}$ \\
\hline \multirow[t]{3}{*}{$4 f^{14} 5 d^{2} D_{5 / 2}$} & $4 f^{14} 6 p^{2} P_{3 / 2}^{\circ}$ & $2773.36^{\mathrm{A}}$ & $2772.54^{a}$ & $0.922^{\mathrm{A}}$ & $0.771^{a}$ & $8.00 \times 10^{8 \mathrm{~A}}$ & $6.69 \times 10^{8 a}$ \\
\hline & & $2773.35^{\mathrm{B}}$ & $2772.550^{b}$ & $1.025^{\mathrm{B}}$ & & $8.89 \times 10^{8 \mathrm{~B}}$ & $8.64 \times 10^{8 c}$ \\
\hline & & & & & & & $\begin{array}{l}7.52 \times 10^{8 d} \\
6.92 \times 10^{8 e}\end{array}$ \\
\hline \multirow[t]{3}{*}{$4 f^{14} 6 p^{2} P_{1 / 2}^{\circ}$} & $4 f^{14} 6 d^{2} D_{3 / 2}$ & $1854.56^{\mathrm{A}}$ & $1854.57^{a}$ & $2.195^{\mathrm{A}}$ & $2.187^{a}$ & $4.26 \times 10^{9 \mathrm{~A}}$ & $4.24 \times 10^{9 a}$ \\
\hline & & $1854.57^{\mathrm{B}}$ & $1854.574^{b}$ & $2.397^{\mathrm{B}}$ & & $4.65 \times 10^{9 \mathrm{~B}}$ & $3.98 \times 10^{9 c}$ \\
\hline & & & & & & & $\begin{array}{l}3.58 \times 10^{9 d} \\
3.37 \times 10^{9 e}\end{array}$ \\
\hline \multirow[t]{3}{*}{$4 f^{14} 6 p^{2} P_{3 / 2}^{\circ}$} & $4 f^{14} 6 d^{2} D_{3 / 2}$ & $2100.12^{\mathrm{A}, \mathrm{B}}$ & $2099.45^{a}$ & $0.388^{\mathrm{A}}$ & $0.386^{a}$ & $5.87 \times 10^{8 \mathrm{~A}}$ & $5.84 \times 10^{8 a}$ \\
\hline & & & $2099.442^{b}$ & $0.423^{\mathrm{B}}$ & & $6.40 \times 10^{8 \mathrm{~B}}$ & $6.88 \times 10^{8 c}$ \\
\hline & & & & & & & $6.12 \times 10^{8 d}$ \\
\hline \multirow[t]{3}{*}{$4 f^{14} 6 p^{2} P_{3 / 2}^{\circ}$} & $4 f^{14} 6 d^{2} D_{5 / 2}$ & $2066.01^{\mathrm{A}}$ & $2065.35^{a}$ & $3.535^{\mathrm{A}}$ & $3.533^{a}$ & $5.52 \times 10^{9 \mathrm{~A}}$ & $\begin{array}{l}5.72 \times 10^{\circ} \\
5.52 \times 10^{9 a}\end{array}$ \\
\hline & & $2066.02^{\mathrm{B}}$ & $2065.346^{b}$ & $3.873^{\mathrm{B}}$ & & $6.05 \times 10^{9 \mathrm{~A}}$ & $6.30 \times 10^{9 c}$ \\
\hline & & & & & & & $\begin{array}{l}5.63 \times 10^{9 d} \\
5.28 \times 10^{9 e}\end{array}$ \\
\hline \multirow[t]{3}{*}{$4 f^{14} 6 p^{2} P_{1 / 2}^{\circ}$} & $4 f^{14} 7 s^{2} S_{1 / 2}$ & $2071.22^{\mathrm{A}}$ & $2070.56^{a}$ & $0.469^{\mathrm{A}}$ & $0.496^{a}$ & $7.29 \times 10^{8 \mathrm{~A}}$ & $7.70 \times 10^{8 a}$ \\
\hline & & $2071.23^{\mathrm{B}}$ & $2070.563^{b}$ & $0.487^{\mathrm{B}}$ & & $7.58 \times 10^{8 \mathrm{~B}}$ & $5.58 \times 10^{8 c}$ \\
\hline & & & & & & & $\begin{array}{l}6.18 \times 10^{8 d} \\
6.38 \times 10^{8 e}\end{array}$ \\
\hline \multirow[t]{4}{*}{$4 f^{14} 6 p^{2} P_{3 / 2}^{\circ}$} & $4 f^{14} 7 s^{2} S_{1 / 2}$ & $2382.31^{\mathrm{A}}$ & $2381.59^{a}$ & $0.799^{\mathrm{A}}$ & $0.862^{a}$ & $9.39 \times 10^{8 \mathrm{~A}}$ & $1.01 \times 10^{9 a}$ \\
\hline & & $2382.32^{\mathrm{B}}$ & $2381.604^{b}$ & $0.847^{\mathrm{B}}$ & & $9.96 \times 10^{8 \mathrm{~B}}$ & $1.13 \times 10^{9 c}$ \\
\hline & & & & & & & $1.14 \times 10^{9 d}$ \\
\hline & & & & & & & $1.16 \times 10^{9 e}$ \\
\hline
\end{tabular}


TABLE I (cont.)

\begin{tabular}{|c|c|c|c|c|c|c|c|}
\hline \multicolumn{2}{|c|}{ Transition } & \multicolumn{2}{|c|}{$\lambda[\AA]$} & \multicolumn{2}{|c|}{$\overline{g f}$} & \multicolumn{2}{|c|}{$g A_{k i}\left[\mathrm{~s}^{-1}\right]$} \\
\hline Lower level & Upper level & This work & Others & This $\mathrm{w}$. & Others & This work & Others \\
\hline $4 f^{14} 6 p^{2} P_{1 / 2}^{\circ}$ & $4 f^{14} 7 d^{2} D_{3 / 2}$ & $1187.34^{\mathrm{A}, \mathrm{B}}$ & $\begin{array}{l}1187.34^{a} \\
1187.340^{b}\end{array}$ & $\begin{array}{l}0.100^{\mathrm{A}} \\
0.263^{\mathrm{B}}\end{array}$ & $0.204^{a}$ & $\begin{array}{l}4.74 \times 10^{8 \mathrm{~A}} \\
1.24 \times 10^{9 \mathrm{~B}}\end{array}$ & $\begin{array}{l}9.64 \times 10^{8 a} \\
1.24 \times 10^{9 c} \\
9.56 \times 10^{8 d} \\
8.04 \times 10^{8 e}\end{array}$ \\
\hline $4 f^{14} 6 p^{2} P_{3 / 2}^{\circ}$ & $4 f^{14} 7 d^{2} D_{3 / 2}$ & $1283.41^{\mathrm{A}, \mathrm{B}}$ & $\begin{array}{l}1283.41^{a} \\
1283.411^{b}\end{array}$ & $\begin{array}{l}0.031^{\mathrm{A}} \\
0.049^{\mathrm{B}}\end{array}$ & $0.038^{a}$ & $\begin{array}{l}1.27 \times 10^{8 \mathrm{~A}} \\
1.97 \times 10^{8 \mathrm{~B}}\end{array}$ & $\begin{array}{l}1.53 \times 10^{8 a} \\
1.81 \times 10^{8 c} \\
1.33 \times 10^{8 d} \\
1.08 \times 10^{8 e}\end{array}$ \\
\hline $4 f^{14} 6 p^{2} P_{3 / 2}^{\circ}$ & $4 f^{14} 7 d^{2} D_{5 / 2}$ & $1277.53^{\mathrm{A}, \mathrm{B}}$ & $\begin{array}{l}1277.53^{a} \\
1277.534^{b}\end{array}$ & $\begin{array}{l}0.297^{\mathrm{A}} \\
0.439^{\mathrm{B}}\end{array}$ & $0.341^{a}$ & $\begin{array}{l}1.22 \times 10^{9 \mathrm{~A}} \\
1.80 \times 10^{9 \mathrm{~B}}\end{array}$ & $\begin{array}{l}1.39 \times 10^{9 a} \\
1.75 \times 10^{9 c} \\
1.31 \times 10^{9 d} \\
1.09 \times 10^{9 e}\end{array}$ \\
\hline $4 f^{14} 6 p^{2} P_{1 / 2}^{\circ}$ & $4 f^{14} 8 s^{2} S_{1 / 2}$ & $1228.74^{\mathrm{A}, \mathrm{B}}$ & $1228.74^{a}$ & $\begin{array}{l}0.073^{\mathrm{A}} \\
0.061^{\mathrm{B}}\end{array}$ & $0.065^{a}$ & $\begin{array}{l}3.23 \times 10^{8 \mathrm{~A}} \\
2.69 \times 10^{8 \mathrm{~B}}\end{array}$ & $\begin{array}{l}2.86 \times 10^{8 a} \\
2.38 \times 10^{8 c} \\
2.58 \times 10^{8 d} \\
2.68 \times 10^{8 e}\end{array}$ \\
\hline $4 f^{14} 6 p^{2} P_{3 / 2}^{\circ}$ & $4 f^{14} 8 s^{2} S_{1 / 2}$ & $1331.92^{\mathrm{A}, \mathrm{B}}$ & $\begin{array}{l}1331.92^{a} \\
1331.929^{b}\end{array}$ & $\begin{array}{l}0.115^{\mathrm{A}} \\
0.112^{\mathrm{B}}\end{array}$ & $0.120^{a}$ & $\begin{array}{l}4.31 \times 10^{8 \mathrm{~A}} \\
4.22 \times 10^{8 \mathrm{~B}}\end{array}$ & $\begin{array}{l}4.49 \times 10^{8 a} \\
4.38 \times 10^{8 c} \\
4.48 \times 10^{8 d} \\
4.52 \times 10^{8 e}\end{array}$ \\
\hline $4 f^{14} 6 p^{2} P_{1 / 2}^{\circ}$ & $4 f^{14} 8 d^{2} D_{3 / 2}$ & $\begin{array}{l}1001.73^{\mathrm{A}} \\
1006.30^{\mathrm{B}}\end{array}$ & - & $\begin{array}{l}0.120^{\mathrm{A}} \\
0.085^{\mathrm{B}}\end{array}$ & - & $\begin{array}{l}8.00 \times 10^{8 \mathrm{~A}} \\
5.60 \times 10^{8 \mathrm{~B}}\end{array}$ & $\begin{array}{l}5.76 \times 10^{8 c} \\
4.04 \times 10^{8 d} \\
3.18 \times 10^{8 e}\end{array}$ \\
\hline $4 f^{14} 6 p^{2} P_{3 / 2}^{\circ}$ & $4 f^{14} 8 d^{2} D_{3 / 2}$ & $\begin{array}{l}1069.26^{\mathrm{A}} \\
1074.47^{\mathrm{B}}\end{array}$ & - & $\begin{array}{l}0.178^{\mathrm{A}} \\
0.016^{\mathrm{B}}\end{array}$ & - & $\begin{array}{l}1.04 \times 10^{9 \mathrm{~A}} \\
9.19 \times 10^{7 \mathrm{~B}}\end{array}$ & $\begin{array}{l}7.96 \times 10^{7 c} \\
5.08 \times 10^{7 d} \\
3.75 \times 10^{7 e}\end{array}$ \\
\hline $4 f^{14} 6 p^{2} P_{3 / 2}^{\circ}$ & $4 f^{14} 8 d^{2} D_{5 / 2}$ & $\begin{array}{l}1065.87^{\mathrm{A}} \\
1072.42^{\mathrm{B}}\end{array}$ & - & $\begin{array}{l}0.424^{\mathrm{A}} \\
0.143^{\mathrm{B}}\end{array}$ & - & $\begin{array}{l}2.49 \times 10^{9 \mathrm{~A}} \\
8.32 \times 10^{8 \mathrm{~B}}\end{array}$ & $\begin{array}{l}7.86 \times 10^{8 c} \\
5.20 \times 10^{8 d} \\
3.95 \times 10^{8 e}\end{array}$ \\
\hline $4 f^{14} 6 p^{2} P_{1 / 2}^{\circ}$ & $4 f^{14} 9 s^{2} S_{1 / 2}$ & $1022.40^{\mathrm{A}, \mathrm{B}}$ & $\begin{array}{l}1022.40^{a} \\
1022.396^{b}\end{array}$ & $\begin{array}{l}0.005^{\mathrm{A}} \\
0.021^{\mathrm{B}}\end{array}$ & $0.023^{a}$ & $\begin{array}{l}2.98 \times 10^{7 \mathrm{~A}} \\
1.37 \times 10^{8 \mathrm{~B}}\end{array}$ & $\begin{array}{l}1.49 \times 10^{8 a} \\
1.23 \times 10^{8 c} \\
1.35 \times 10^{8 d} \\
1.44 \times 10^{8 e}\end{array}$ \\
\hline $4 f^{14} 6 p^{2} P_{3 / 2}^{\circ}$ & $4 f^{14} 9 s^{2} S_{1 / 2}$ & $1092.84^{\mathrm{A}, \mathrm{B}}$ & $\begin{array}{l}1092.84^{a} \\
1092.842^{b}\end{array}$ & $\begin{array}{l}0.112^{\mathrm{A}} \\
0.040^{\mathrm{B}}\end{array}$ & $0.044^{a}$ & $\begin{array}{l}6.24 \times 10^{8 \mathrm{~A}} \\
2.24 \times 10^{8 \mathrm{~B}}\end{array}$ & $\begin{array}{l}2.43 \times 10^{8 a} \\
2.28 \times 10^{8 c} \\
2.32 \times 10^{8 d} \\
2.36 \times 10^{8 e}\end{array}$ \\
\hline $4 f^{14} 6 p^{2} P_{1 / 2}^{\circ}$ & $4 f^{14} 9 d^{2} D_{3 / 2}$ & $\begin{array}{l}925.12^{\mathrm{A}} \\
925.65^{\mathrm{B}}\end{array}$ & - & $\begin{array}{l}0.057^{\mathrm{A}} \\
0.039^{\mathrm{B}}\end{array}$ & - & $\begin{array}{l}4.45 \times 10^{8 \mathrm{~A}} \\
3.07 \times 10^{8 \mathrm{~B}}\end{array}$ & $\begin{array}{l}1.23 \times 10^{8 c} \\
2.12 \times 10^{8 d} \\
1.60 \times 10^{8 e}\end{array}$ \\
\hline $4 f^{14} 6 p^{2} P_{3 / 2}^{\circ}$ & $4 f^{14} 9 d^{2} D_{3 / 2}$ & $\begin{array}{l}982.42^{\mathrm{A}} \\
983.02^{\mathrm{B}}\end{array}$ & - & $\begin{array}{l}0.014^{\mathrm{A}} \\
0.007^{\mathrm{B}}\end{array}$ & - & $\begin{array}{l}9.60 \times 10^{7 \mathrm{~A}} \\
5.13 \times 10^{7 \mathrm{~B}}\end{array}$ & $\begin{array}{l}4.32 \times 10^{7 c} \\
2.52 \times 10^{7 d} \\
1.72 \times 10^{7 e}\end{array}$ \\
\hline $4 f^{14} 6 p^{2} P_{3 / 2}^{\circ}$ & $4 f^{14} 9 d^{2} D_{5 / 2}$ & $\begin{array}{l}981.80^{\mathrm{A}} \\
981.98^{\mathrm{B}}\end{array}$ & - & $\begin{array}{l}0.087^{\mathrm{A}} \\
0.067^{\mathrm{B}}\end{array}$ & - & $\begin{array}{l}6.06 \times 10^{8 \mathrm{~A}} \\
4.63 \times 10^{8 \mathrm{~B}}\end{array}$ & $\begin{array}{l}4.31 \times 10^{8 c} \\
2.64 \times 10^{8 d} \\
1.87 \times 10^{8 e}\end{array}$ \\
\hline $4 f^{14} 6 p^{2} P_{1 / 2}^{\circ}$ & $4 f^{14} 10 s^{2} S_{1 / 2}$ & $932.95^{\mathrm{A}, \mathrm{B}}$ & $932.95^{a}$ & $0.010^{\mathrm{A}, \mathrm{B}}$ & $0.012^{a}$ & $\begin{array}{l}7.34 \times 10^{7 \mathrm{~A}} \\
8.04 \times 10^{7 \mathrm{~B}}\end{array}$ & $\begin{array}{l}8.81 \times 10^{7 a} \\
7.34 \times 10^{7 c} \\
8.10 \times 10^{7 d} \\
8.64 \times 10^{7 e}\end{array}$ \\
\hline
\end{tabular}


TABLE I (cont.)

\begin{tabular}{|c|c|c|c|c|c|c|c|}
\hline \multicolumn{2}{|c|}{ Transition } & \multicolumn{2}{|c|}{$\lambda[\AA]$} & \multicolumn{2}{|c|}{$g f$} & \multicolumn{2}{|c|}{$g A_{k i}\left[\mathrm{~s}^{-1}\right]$} \\
\hline Lower level & Upper level & This work & Others & This w. & Others & This work & Others \\
\hline $4 f^{14} 6 p^{2} P_{3 / 2}^{\circ}$ & $4 f^{14} 10 s^{2} S_{1 / 2}$ & $\begin{array}{l}1001.67^{\mathrm{A}} \\
991.26^{\mathrm{B}}\end{array}$ & $\begin{array}{l}991.26^{a} \\
991.256^{b}\end{array}$ & $0.020^{\mathrm{A}, \mathrm{B}}$ & $0.022^{a}$ & $\begin{array}{l}1.30 \times 10^{8 \mathrm{~A}} \\
1.34 \times 10^{8 \mathrm{~B}}\end{array}$ & $\begin{array}{l}1.47 \times 10^{8 a} \\
1.36 \times 10^{8 c} \\
1.38 \times 10^{8 d} \\
1.40 \times 10^{8 e}\end{array}$ \\
\hline $4 f^{14} 6 s^{2} S_{1 / 2}$ & $4 f^{14} 7 p^{2} P_{1 / 2}^{\circ}$ & $996.44^{\mathrm{A}, \mathrm{B}}$ & - & $\begin{array}{l}0.091^{\mathrm{A}} \\
0.003^{\mathrm{B}}\end{array}$ & - & $\begin{array}{l}0.61 \times 10^{7 \mathrm{~A}} \\
1.76 \times 10^{7 \mathrm{~B}}\end{array}$ & $\begin{array}{l}1.22 \times 10^{6 c} \\
4.50 \times 10^{7 d} \\
9.90 \times 10^{7 e}\end{array}$ \\
\hline $4 f^{14} 6 s^{2} S_{1 / 2}$ & $4 f^{14} 7 p^{2} P_{3 / 2}^{\circ}$ & $972.66^{\mathrm{A}, \mathrm{B}}$ & $\begin{array}{l}972.66^{a} \\
972.662^{b}\end{array}$ & $\begin{array}{l}0.087^{\mathrm{A}} \\
0.005^{\mathrm{B}}\end{array}$ & $0.001^{a}$ & $\begin{array}{l}6.13 \times 10^{8 \mathrm{~A}} \\
3.78 \times 10^{7 \mathrm{~B}}\end{array}$ & $\begin{array}{l}8.75 \times 10^{6 a} \\
7.48 \times 10^{7 c} \\
2.34 \times 10^{5 d} \\
1.79 \times 10^{7 e}\end{array}$ \\
\hline $4 f^{14} 5 d^{2} D_{3 / 2}$ & $4 f^{14} 7 p^{2} P_{1 / 2}^{\circ}$ & $1056.53^{\mathrm{A}, \mathrm{B}}$ & $\begin{array}{l}1056.53^{a} \\
1056.529^{b}\end{array}$ & $\begin{array}{l}0.050^{\mathrm{A}} \\
0.033^{\mathrm{B}}\end{array}$ & $0.017^{a}$ & $\begin{array}{l}2.99 \times 10^{8 \mathrm{~A}} \\
1.99 \times 10^{8 \mathrm{~B}}\end{array}$ & $\begin{array}{l}9.88 \times 10^{7 a} \\
1.11 \times 10^{8 c} \\
4.88 \times 10^{7 d} \\
2.52 \times 10^{7 e}\end{array}$ \\
\hline $4 f^{14} 5 d^{2} D_{3 / 2}$ & $4 f^{14} 7 p^{2} P_{3 / 2}^{\circ}$ & $1029.83^{\mathrm{A}, \mathrm{B}}$ & $\begin{array}{l}1029.83^{a} \\
1029.833^{b}\end{array}$ & $\begin{array}{l}0.008^{\mathrm{A}} \\
0.007^{\mathrm{B}}\end{array}$ & $0.003^{a}$ & $\begin{array}{l}5.32 \times 10^{7 \mathrm{~A}} \\
4.30 \times 10^{7 \mathrm{~B}}\end{array}$ & $\begin{array}{l}2.13 \times 10^{7 a} \\
3.63 \times 10^{7 c} \\
2.42 \times 10^{7 d} \\
1.86 \times 10^{7 e}\end{array}$ \\
\hline $4 f^{14} 5 d^{2} D_{5 / 2}$ & $4 f^{14} 7 p^{2} P_{3 / 2}^{\circ}$ & $1061.99^{\mathrm{A}, \mathrm{B}}$ & $\begin{array}{l}1061.99^{a} \\
1061.986^{b}\end{array}$ & $\begin{array}{l}0.104^{\mathrm{A}} \\
0.060^{\mathrm{B}}\end{array}$ & $0.030^{a}$ & $\begin{array}{l}6.17 \times 10^{8 \mathrm{~A}} \\
3.53 \times 10^{8 \mathrm{~B}}\end{array}$ & $\begin{array}{l}1.75 \times 10^{8 a} \\
2.74 \times 10^{8 c} \\
1.70 \times 10^{8 d} \\
1.23 \times 10^{8 e}\end{array}$ \\
\hline $4 f^{14} 7 s^{2} S_{1 / 2}$ & $4 f^{14} 7 p^{2} P_{1 / 2}^{\circ}$ & $\begin{array}{l}7311.97^{\mathrm{A}} \\
7312.13^{\mathrm{B}}\end{array}$ & $\begin{array}{l}7310.13^{a} \\
7310.252^{b}\end{array}$ & $\begin{array}{l}0.749^{\mathrm{A}} \\
1.044^{\mathrm{B}}\end{array}$ & $0.977^{a}$ & $\begin{array}{l}0.93 \times 10^{8 \mathrm{~A}} \\
1.30 \times 10^{8 \mathrm{~B}}\end{array}$ & $\begin{array}{l}1.22 \times 10^{8 a} \\
1.32 \times 10^{8 c} \\
1.28 \times 10^{8 d} \\
1.26 \times 10^{8 e}\end{array}$ \\
\hline $4 f^{14} 7 s^{2} S_{1 / 2}$ & $4 f^{14} 7 p^{2} P_{3 / 2}^{\circ}$ & $\begin{array}{l}6199.79^{\mathrm{A}} \\
6199.78^{\mathrm{B}}\end{array}$ & $\begin{array}{l}6198.06^{a} \\
6198.128^{b}\end{array}$ & $\begin{array}{l}2.201^{\mathrm{A}} \\
2.462^{\mathrm{B}}\end{array}$ & $2.305^{a}$ & $\begin{array}{l}3.82 \times 10^{8 \mathrm{~A}} \\
4.27 \times 10^{8 \mathrm{~B}}\end{array}$ & $\begin{array}{l}4.00 \times 10^{8 a, c} \\
3.90 \times 10^{8 d} \\
3.83 \times 10^{8 e}\end{array}$ \\
\hline $4 f^{14} 6 d^{2} D_{3 / 2}$ & $4 f^{14} 7 p^{2} P_{1 / 2}^{\circ}$ & $\begin{array}{l}12444.27^{\mathrm{A}} \\
12444.78^{\mathrm{B}}\end{array}$ & - & $\begin{array}{l}0.593^{\mathrm{A}} \\
0.823^{\mathrm{B}}\end{array}$ & - & $\begin{array}{l}2.55 \times 10^{7 \mathrm{~A}} \\
3.55 \times 10^{7 \mathrm{~B}}\end{array}$ & $\begin{array}{l}3.70 \times 10^{7 c} \\
3.90 \times 10^{7 d} \\
4.04 \times 10^{7 e}\end{array}$ \\
\hline $4 f^{14} 6 d^{2} D_{3 / 2}$ & $4 f^{14} 7 p^{2} P_{3 / 2}^{\circ}$ & $\begin{array}{l}9533.60^{\mathrm{A}} \\
9533.62^{\mathrm{B}}\end{array}$ & $9530.98^{a}$ & $\begin{array}{l}0.192^{\mathrm{A}} \\
0.215^{\mathrm{B}}\end{array}$ & $0.202^{a}$ & $\begin{array}{l}1.41 \times 10^{7 \mathrm{~A}} \\
1.58 \times 10^{7 \mathrm{~B}}\end{array}$ & $\begin{array}{l}1.49 \times 10^{7 a} \\
1.42 \times 10^{7 c} \\
1.48 \times 10^{7 d} \\
1.53 \times 10^{7 e}\end{array}$ \\
\hline $4 f^{14} 6 d^{2} D_{5 / 2}$ & $4 f^{14} 7 p^{2} P_{3 / 2}^{\circ}$ & $\begin{array}{l}10305.91^{\mathrm{A}} \\
10305.88^{\mathrm{B}}\end{array}$ & - & $\begin{array}{l}1.594^{\mathrm{A}} \\
1.789^{\mathrm{B}}\end{array}$ & - & $\begin{array}{l}1.00 \times 10^{8 \mathrm{~A}} \\
1.12 \times 10^{8 \mathrm{~B}}\end{array}$ & $\begin{array}{l}1.09 \times 10^{8 c} \\
1.14 \times 10^{8 d} \\
1.15 \times 10^{8 e}\end{array}$ \\
\hline $4 f^{14} 7 p^{2} P_{1 / 2}^{\circ}$ & $4 f^{14} 7 d^{2} D_{3 / 2}$ & $\begin{array}{l}4491.34^{\mathrm{A}} \\
4491.27^{\mathrm{B}}\end{array}$ & $\begin{array}{l}4490.01^{a} \\
4490.004^{b}\end{array}$ & $\begin{array}{l}2.029^{\mathrm{A}} \\
2.708^{\mathrm{B}}\end{array}$ & $2.676^{a}$ & $\begin{array}{l}6.71 \times 10^{8 \mathrm{~A}} \\
8.96 \times 10^{8 \mathrm{~B}}\end{array}$ & $\begin{array}{l}8.85 \times 10^{8 a} \\
7.36 \times 10^{8 c} \\
7.28 \times 10^{8 d} \\
7.24 \times 10^{8 e}\end{array}$ \\
\hline $4 f^{14} 7 p^{2} P_{3 / 2}^{\circ}$ & $4 f^{14} 7 d^{2} D_{3 / 2}$ & $5047.52^{\mathrm{A}, \mathrm{B}}$ & $\begin{array}{l}5046.12^{a} \\
5046.122^{b}\end{array}$ & $\begin{array}{l}0.421^{\mathrm{A}} \\
0.482^{\mathrm{B}}\end{array}$ & $0.476^{a}$ & $\begin{array}{l}1.10 \times 10^{8 \mathrm{~A}} \\
1.26 \times 10^{8 \mathrm{~B}}\end{array}$ & $\begin{array}{l}1.25 \times 10^{8 a} \\
1.35 \times 10^{8 c} \\
1.32 \times 10^{8 d} \\
1.30 \times 10^{8 e}\end{array}$ \\
\hline $4 f^{14} 7 p^{2} P_{3 / 2}^{\circ}$ & $4 f^{14} 7 d^{2} D_{5 / 2}$ & $4957.81^{\mathrm{A}, \mathrm{B}}$ & $\begin{array}{l}4956.43^{a} \\
4956.430^{b}\end{array}$ & $\begin{array}{l}4.265^{\mathrm{A}} \\
4.416^{\mathrm{B}}\end{array}$ & $4.364^{a}$ & $\begin{array}{l}1.16 \times 10^{9 \mathrm{~A}} \\
1.20 \times 10^{9 \mathrm{~B}}\end{array}$ & $\begin{array}{l}1.18 \times 10^{9 a} \\
1.22 \times 10^{9 c} \\
1.21 \times 10^{9 d} \\
1.19 \times 10^{9 e}\end{array}$ \\
\hline
\end{tabular}


TABLE I (cont.)

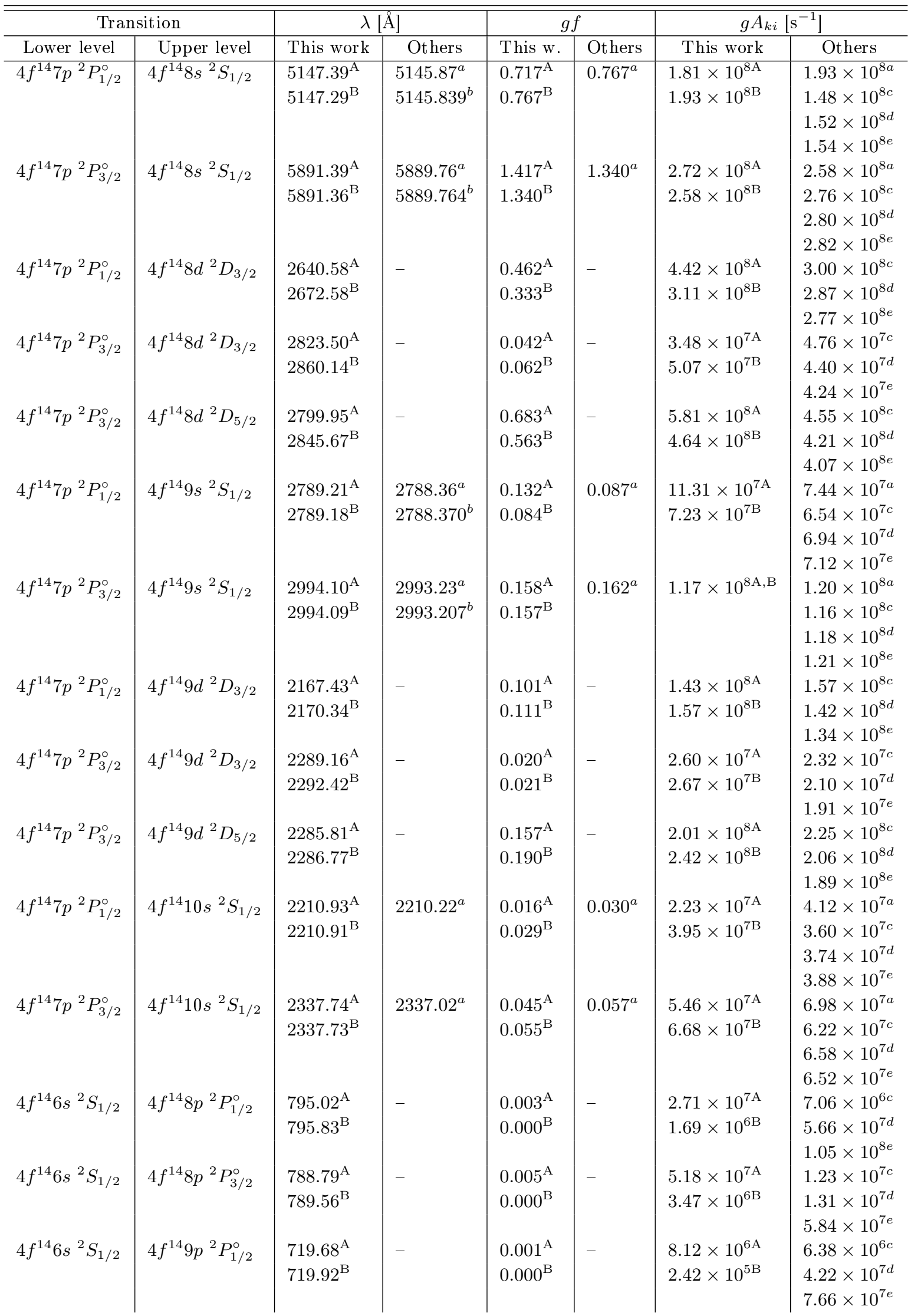


TABLE I (cont.)

\begin{tabular}{|c|c|c|c|c|c|c|c|}
\hline \multicolumn{2}{|c|}{ Transition } & \multicolumn{2}{|c|}{$\lambda[\AA]$} & \multicolumn{2}{|c|}{$g f$} & \multicolumn{2}{|c|}{$g A_{k i}\left[\mathrm{~s}^{-1}\right]$} \\
\hline Lower level & Upper level & This work & Others & This w. & Others & This work & Others \\
\hline $4 f^{14} 6 s^{2} S_{1 / 2}$ & $4 f^{14} 9 p^{2} P_{3 / 2}^{\circ}$ & $\begin{array}{l}716.74^{\mathrm{A}} \\
716.98^{\mathrm{B}}\end{array}$ & - & $\begin{array}{l}0.001^{\mathrm{A}} \\
0.000^{\mathrm{B}}\end{array}$ & - & $\begin{array}{l}1.59 \times 10^{7 \mathrm{~A}} \\
4.90 \times 10^{5 \mathrm{~B}}\end{array}$ & $\begin{array}{l}3.63 \times 10^{6 c} \\
1.56 \times 10^{7 d} \\
5.28 \times 10^{7 e}\end{array}$ \\
\hline $4 f^{14} 6 s^{2} S_{1 / 2}$ & $4 f^{14} 10 p^{2} P_{1 / 2}^{\circ}$ & $\begin{array}{l}681.39^{\mathrm{A}} \\
681.50^{\mathrm{B}}\end{array}$ & - & $0.000^{\mathrm{A}, \mathrm{B}}$ & - & $\begin{array}{l}4.44 \times 10^{6 \mathrm{~A}} \\
3.61 \times 10^{4 \mathrm{~B}}\end{array}$ & $\begin{array}{l}4.86 \times 10^{6 c} \\
2.98 \times 10^{7 d} \\
5.38 \times 10^{7 e}\end{array}$ \\
\hline $4 f^{14} 6 s^{2} S_{1 / 2}$ & $4 f^{14} 10 p^{2} P_{3 / 2}^{\circ}$ & $\begin{array}{l}679.74^{\mathrm{A}} \\
679.85^{\mathrm{B}}\end{array}$ & - & $\begin{array}{l}0.001^{\mathrm{A}} \\
0.000^{\mathrm{B}}\end{array}$ & - & $\begin{array}{l}8.74 \times 10^{6 \mathrm{~A}} \\
7.27 \times 10^{4 \mathrm{~B}}\end{array}$ & $\begin{array}{l}1.43 \times 10^{6 c} \\
1.33 \times 10^{7 d} \\
4.07 \times 10^{7 e}\end{array}$ \\
\hline $4 f^{14} 7 s^{2} S_{1 / 2}$ & $4 f^{14} 8 p^{2} P_{1 / 2}^{\circ}$ & $\begin{array}{l}2557.45^{\mathrm{A}} \\
2565.86^{\mathrm{B}}\end{array}$ & - & $\begin{array}{l}0.007^{\mathrm{A}} \\
0.005^{\mathrm{B}}\end{array}$ & - & $\begin{array}{l}6.91 \times 10^{6 \mathrm{~A}} \\
5.41 \times 10^{6 \mathrm{~B}}\end{array}$ & $\begin{array}{l}7.68 \times 10^{4 c} \\
1.93 \times 10^{5 d} \\
6.84 \times 10^{5 e}\end{array}$ \\
\hline $4 f^{14} 7 s^{2} S_{1 / 2}$ & $4 f^{14} 8 p^{2} P_{3 / 2}^{\circ}$ & $\begin{array}{l}2494.06^{\mathrm{A}} \\
2501.75^{\mathrm{B}}\end{array}$ & - & $\begin{array}{l}0.014^{\mathrm{A}} \\
0.011^{\mathrm{B}}\end{array}$ & - & $\begin{array}{l}1.50 \times 10^{7 \mathrm{~A}} \\
1.17 \times 10^{7 \mathrm{~B}}\end{array}$ & $\begin{array}{l}2.26 \times 10^{7 c} \\
1.42 \times 10^{7 d} \\
1.05 \times 10^{7 e}\end{array}$ \\
\hline $4 f^{14} 7 s^{2} S_{1 / 2}$ & $4 f^{14} 9 p^{2} P_{1 / 2}^{\circ}$ & $\begin{array}{l}1913.14^{\mathrm{A}} \\
1914.89^{\mathrm{B}}\end{array}$ & - & $\begin{array}{l}0.001^{\mathrm{A}} \\
0.000^{\mathrm{B}}\end{array}$ & - & $\begin{array}{l}1.09 \times 10^{6 \mathrm{~A}} \\
7.51 \times 10^{5 \mathrm{~B}}\end{array}$ & $\begin{array}{l}8.54 \times 10^{5 c} \\
2.44 \times 10^{6 d} \\
4.12 \times 10^{6 e}\end{array}$ \\
\hline $4 f^{14} 7 s^{2} S_{1 / 2}$ & $4 f^{14} 9 p^{2} P_{3 / 2}^{\circ}$ & $\begin{array}{l}1892.52^{\mathrm{A}} \\
1894.22^{\mathrm{B}}\end{array}$ & - & $0.001^{\mathrm{A}, \mathrm{B}}$ & - & $\begin{array}{l}2.28 \times 10^{6 \mathrm{~A}} \\
1.55 \times 10^{6 \mathrm{~B}}\end{array}$ & $\begin{array}{l}4.00 \times 10^{6 c} \\
1.30 \times 10^{6 d} \\
2.37 \times 10^{5 e}\end{array}$ \\
\hline $4 f^{14} 7 s^{2} S_{1 / 2}$ & $4 f^{14} 10 p^{2} P_{1 / 2}^{\circ}$ & $\begin{array}{l}1664.51^{\mathrm{A}} \\
1665.19^{\mathrm{B}}\end{array}$ & - & $0.000^{\mathrm{A}, \mathrm{B}}$ & - & $\begin{array}{l}3.27 \times 10^{5 \mathrm{~A}} \\
1.52 \times 10^{5 \mathrm{~B}}\end{array}$ & $\begin{array}{l}8.84 \times 10^{5 c} \\
2.62 \times 10^{6 d} \\
3.98 \times 10^{6 e}\end{array}$ \\
\hline $4 f^{14} 7 s^{2} S_{1 / 2}$ & $4 f^{14} 10 p^{2} P_{3 / 2}^{\circ}$ & $\begin{array}{l}1654.71^{\mathrm{A}} \\
1655.37^{\mathrm{B}}\end{array}$ & - & $0.000^{\mathrm{A}, \mathrm{B}}$ & - & $\begin{array}{l}6.73 \times 10^{5 \mathrm{~A}} \\
3.10 \times 10^{5 \mathrm{~B}}\end{array}$ & $\begin{array}{l}1.56 \times 10^{6 c} \\
1.14 \times 10^{5 d} \\
3.80 \times 10^{4 e}\end{array}$ \\
\hline $4 f^{14} 5 d^{2} D_{3 / 2}$ & $4 f^{14} 5 f^{2} F_{5 / 2}^{\circ}$ & $\begin{array}{l}1001.79^{\mathrm{A}} \\
1001.17^{\mathrm{B}}\end{array}$ & $\begin{array}{l}1001.17^{a} \\
1001.175^{b}\end{array}$ & $\begin{array}{l}2.107^{\mathrm{A}} \\
1.873^{\mathrm{B}}\end{array}$ & $1.283^{a}$ & $\begin{array}{l}1.40 \times 10^{10 A} \\
1.25 \times 10^{10 B}\end{array}$ & $\begin{array}{l}8.53 \times 10^{9 a} \\
1.21 \times 10^{10 c} \\
9.42 \times 10^{9 d} \\
8.10 \times 10^{9 e}\end{array}$ \\
\hline $4 f^{14} 5 d^{2} D_{5 / 2}$ & $4 f^{14} 5 f^{2} F_{5 / 2}^{\circ}$ & $\begin{array}{l}1032.20^{\mathrm{A}} \\
1031.54^{\mathrm{B}}\end{array}$ & $\begin{array}{l}1031.54^{a} \\
1031.537^{b}\end{array}$ & $\begin{array}{l}0.160^{\mathrm{A}} \\
0.130^{\mathrm{B}}\end{array}$ & $0.089^{a}$ & $\begin{array}{l}9.99 \times 10^{8 \mathrm{~A}} \\
8.14 \times 10^{8 \mathrm{~B}}\end{array}$ & $\begin{array}{l}5.57 \times 10^{8 a} \\
8.64 \times 10^{8 c} \\
6.78 \times 10^{8 d} \\
5.84 \times 10^{8 e}\end{array}$ \\
\hline $4 f^{14} 5 d^{2} D_{5 / 2}$ & $4 f^{14} 5 f^{2} F_{7 / 2}^{\circ}$ & $\begin{array}{l}1029.66^{\mathrm{A}} \\
1030.33^{\mathrm{B}}\end{array}$ & $\begin{array}{l}1030.33^{a} \\
1030.332^{b}\end{array}$ & $\begin{array}{l}3.053^{\mathrm{A}} \\
2.601^{\mathrm{B}}\end{array}$ & $1.780^{a}$ & $\begin{array}{l}1.92 \times 10^{10 A} \\
1.63 \times 10^{10 B}\end{array}$ & $\begin{array}{l}1.12 \times 10^{10 a} \\
1.70 \times 10^{10 c} \\
1.34 \times 10^{10 d} \\
1.16 \times 10^{10 e}\end{array}$ \\
\hline $4 f^{14} 5 d^{2} D_{3 / 2}$ & $4 f^{14} 6 f^{2} F_{5 / 2}^{\circ}$ & $\begin{array}{l}811.58^{\mathrm{A}} \\
810.73^{\mathrm{B}}\end{array}$ & $\begin{array}{l}810.73^{a} \\
810.728^{b}\end{array}$ & $\begin{array}{l}0.787^{\mathrm{A}} \\
0.680^{\mathrm{B}}\end{array}$ & $0.411^{a}$ & $\begin{array}{l}7.97 \times 10^{9 \mathrm{~A}} \\
6.90 \times 10^{9 \mathrm{~B}}\end{array}$ & $\begin{array}{l}4.16 \times 10^{9 a} \\
6.72 \times 10^{9 c} \\
4.76 \times 10^{9 d} \\
3.86 \times 10^{9 e}\end{array}$ \\
\hline $4 f^{14} 5 d^{2} D_{5 / 2}$ & $4 f^{14} 6 f^{2} F_{5 / 2}^{\circ}$ & $\begin{array}{l}831.42^{\mathrm{A}} \\
830.53^{\mathrm{B}}\end{array}$ & $\begin{array}{l}830.53^{a} \\
830.530^{b}\end{array}$ & $\begin{array}{l}0.056^{\mathrm{A}} \\
0.047^{\mathrm{B}}\end{array}$ & $0.029^{a}$ & $\begin{array}{l}5.42 \times 10^{8 \mathrm{~A}} \\
4.58 \times 10^{8 \mathrm{~B}}\end{array}$ & $\begin{array}{l}2.76 \times 10^{8 a} \\
4.70 \times 10^{8 c} \\
3.34 \times 10^{8 d} \\
2.71 \times 10^{8 e}\end{array}$ \\
\hline $4 f^{14} 5 d^{2} D_{5 / 2}$ & $4 f^{14} 6 f^{2} F_{7 / 2}^{\circ}$ & $\begin{array}{l}831.38^{\mathrm{A}} \\
832.28^{\mathrm{B}}\end{array}$ & $\begin{array}{l}832.28^{a} \\
832.279^{b}\end{array}$ & $\begin{array}{l}1.109^{\mathrm{A}} \\
0.946^{\mathrm{B}}\end{array}$ & $0.573^{a}$ & $\begin{array}{l}10.70 \times 10^{9 \mathrm{~A}} \\
9.11 \times 10^{9 \mathrm{~B}}\end{array}$ & $\begin{array}{l}5.53 \times 10^{9 a} \\
9.28 \times 10^{9 c} \\
6.63 \times 10^{9 d} \\
5.41 \times 10^{9 e}\end{array}$ \\
\hline
\end{tabular}


TABLE I (cont.)

\begin{tabular}{|c|c|c|c|c|c|c|c|}
\hline \multicolumn{2}{|c|}{ Transition } & \multicolumn{2}{|c|}{$\lambda[\AA]$} & \multicolumn{2}{|c|}{$g f$} & \multicolumn{2}{|c|}{$g A_{k i}\left[\mathrm{~s}^{-1}\right]$} \\
\hline Lower level & Upper level & This work & Others & This w. & Others & This work & Others \\
\hline $4 f^{14} 5 d^{2} D_{3 / 2}$ & $4 f^{14} 7 f^{2} F_{5 / 2}^{\circ}$ & $738.76^{\mathrm{A}, \mathrm{B}}$ & $\begin{array}{l}738.76^{a} \\
738.757^{b}\end{array}$ & $\begin{array}{l}0.383^{\mathrm{A}} \\
0.328^{\mathrm{B}}\end{array}$ & $0.184^{a}$ & $\begin{array}{l}4.68 \times 10^{9 \mathrm{~A}} \\
4.01 \times 10^{9 \mathrm{~B}}\end{array}$ & $\begin{array}{l}2.24 \times 10^{9 a} \\
3.90 \times 10^{9 c} \\
2.62 \times 10^{9 d} \\
2.04 \times 10^{9 e}\end{array}$ \\
\hline $4 f^{14} 5 d^{2} D_{5 / 2}$ & $4 f^{14} 7 f^{2} F_{5 / 2}^{\circ}$ & $755.16^{\mathrm{A}, \mathrm{B}}$ & $\begin{array}{l}755.16^{a} \\
755.159^{b}\end{array}$ & $\begin{array}{l}0.027^{\mathrm{A}} \\
0.023^{\mathrm{B}}\end{array}$ & $0.013^{a}$ & $\begin{array}{l}3.18 \times 10^{8 \mathrm{~A}} \\
2.69 \times 10^{8 \mathrm{~B}}\end{array}$ & $\begin{array}{l}1.50 \times 10^{8 a} \\
2.72 \times 10^{8 c} \\
1.83 \times 10^{8 d} \\
1.42 \times 10^{8 e}\end{array}$ \\
\hline $4 f^{14} 5 d^{2} D_{5 / 2}$ & $4 f^{14} 7 f^{2} F_{7 / 2}^{\circ}$ & $755.03^{\mathrm{A}, \mathrm{B}}$ & $\begin{array}{l}755.03^{a} \\
755.032^{b}\end{array}$ & $\begin{array}{l}0.540^{\mathrm{A}} \\
0.459^{\mathrm{B}}\end{array}$ & $0.257^{a}$ & $\begin{array}{l}6.31 \times 10^{9 \mathrm{~A}} \\
5.37 \times 10^{9 \mathrm{~B}}\end{array}$ & $\begin{array}{l}3.00 \times 10^{9 a} \\
5.38 \times 10^{9 c} \\
3.65 \times 10^{9 d} \\
2.86 \times 10^{8 e}\end{array}$ \\
\hline $4 f^{14} 5 d^{2} D_{3 / 2}$ & $4 f^{14} 8 f^{2} F_{5 / 2}^{\circ}$ & $700.25^{\mathrm{A}, \mathrm{B}}$ & $\begin{array}{l}700.25^{a} \\
700.250^{b}\end{array}$ & $\begin{array}{l}0.219^{\mathrm{A}} \\
0.187^{\mathrm{B}}\end{array}$ & $0.099^{a}$ & $\begin{array}{l}2.98 \times 10^{9 \mathrm{~A}} \\
2.54 \times 10^{9 \mathrm{~B}}\end{array}$ & $\begin{array}{l}1.35 \times 10^{9 a} \\
2.44 \times 10^{9 c} \\
1.58 \times 10^{9 d} \\
1.20 \times 10^{9 e}\end{array}$ \\
\hline $4 f^{14} 5 d^{2} D_{5 / 2}$ & $4 f^{14} 8 f^{2} F_{5 / 2}^{\circ}$ & $714.97^{\mathrm{A}, \mathrm{B}}$ & $714.97^{a}$ & $\begin{array}{l}0.016^{\mathrm{A}} \\
0.013^{\mathrm{B}}\end{array}$ & $0.007^{a}$ & $\begin{array}{l}2.03 \times 10^{8 \mathrm{~A}} \\
1.70 \times 10^{8 \mathrm{~B}}\end{array}$ & $\begin{array}{l}9.03 \times 10^{7 a} \\
1.70 \times 10^{8 c} \\
1.10 \times 10^{8 d} \\
8.34 \times 10^{7 e}\end{array}$ \\
\hline $4 f^{14} 5 d^{2} D_{5 / 2}$ & $4 f^{14} 8 f^{2} F_{7 / 2}^{\circ}$ & $714.89^{\mathrm{A}, \mathrm{B}}$ & $\begin{array}{l}714.89^{a} \\
714.887^{b}\end{array}$ & $\begin{array}{l}0.309^{\mathrm{A}} \\
0.261^{\mathrm{B}}\end{array}$ & $0.138^{a}$ & $\begin{array}{l}4.03 \times 10^{9 \mathrm{~A}} \\
3.41 \times 10^{9 \mathrm{~B}}\end{array}$ & $\begin{array}{l}1.81 \times 10^{9 a} \\
3.37 \times 10^{9 c} \\
2.21 \times 10^{9 d} \\
1.68 \times 10^{9 e}\end{array}$ \\
\hline $4 f^{14} 5 d^{2} D_{3 / 2}$ & $4 f^{14} 9 f^{2} F_{5 / 2}^{\circ}$ & $677.34^{\mathrm{A}, \mathrm{B}}$ & $\begin{array}{l}677.34^{a} \\
677.342^{b}\end{array}$ & $\begin{array}{l}0.140^{\mathrm{A}} \\
0.117^{\mathrm{B}}\end{array}$ & $0.060^{a}$ & $\begin{array}{l}2.04 \times 10^{9 \mathrm{~A}} \\
1.70 \times 10^{9 \mathrm{~B}}\end{array}$ & $\begin{array}{l}8.71 \times 10^{8 a} \\
1.62 \times 10^{9 c} \\
1.03 \times 10^{9 d} \\
7.68 \times 10^{8 e}\end{array}$ \\
\hline $4 f^{14} 5 d^{2} D_{5 / 2}$ & $4 f^{14} 9 f^{2} F_{5 / 2}^{\circ}$ & $691.11^{\mathrm{A}, \mathrm{B}}$ & $691.11^{a}$ & $\begin{array}{l}0.010^{\mathrm{A}} \\
0.008^{\mathrm{B}}\end{array}$ & $0.004^{a}$ & $\begin{array}{l}1.39 \times 10^{8 \mathrm{~A}} \\
1.15 \times 10^{8 \mathrm{~B}}\end{array}$ & $\begin{array}{l}5.85 \times 10^{7 a} \\
1.12 \times 10^{8 c} \\
7.14 \times 10^{7 d} \\
5.32 \times 10^{7 e}\end{array}$ \\
\hline $4 f^{14} 5 d^{2} D_{5 / 2}$ & $4 f^{14} 9 f^{2} F_{7 / 2}^{\circ}$ & $691.05^{\mathrm{A}, \mathrm{B}}$ & $\begin{array}{l}691.05^{a} \\
691.053^{b}\end{array}$ & $\begin{array}{l}0.198^{\mathrm{A}} \\
0.164^{\mathrm{B}}\end{array}$ & $0.084^{a}$ & $\begin{array}{l}2.76 \times 10^{9 \mathrm{~A}} \\
2.29 \times 10^{9 \mathrm{~B}}\end{array}$ & $\begin{array}{l}1.17 \times 10^{9 a} \\
2.23 \times 10^{9 c} \\
1.43 \times 10^{9 d} \\
1.07 \times 10^{9 e}\end{array}$ \\
\hline $4 f^{14} 6 d^{2} D_{3 / 2}$ & $4 f^{14} 5 f^{2} F_{5 / 2}^{\circ}$ & $\begin{array}{l}7571.77^{\mathrm{A}} \\
7536.31^{\mathrm{B}}\end{array}$ & $\begin{array}{l}7534.29^{a} \\
7534.267^{b}\end{array}$ & $\begin{array}{l}2.762^{\mathrm{A}} \\
2.915^{\mathrm{B}}\end{array}$ & $2.736^{a}$ & $\begin{array}{l}3.21 \times 10^{8 \mathrm{~A}} \\
3.42 \times 10^{8 \mathrm{~B}}\end{array}$ & $\begin{array}{l}3.21 \times 10^{8 a} \\
3.19 \times 10^{8 c} \\
3.26 \times 10^{8 d} \\
3.29 \times 10^{8 e}\end{array}$ \\
\hline $4 f^{14} 6 d^{2} D_{5 / 2}$ & $4 f^{14} 5 f^{2} F_{5 / 2}^{\circ}$ & $\begin{array}{l}8050.94^{\mathrm{A}} \\
8010.83^{\mathrm{B}}\end{array}$ & $\begin{array}{l}8008.69^{a} \\
8008.590^{b}\end{array}$ & $\begin{array}{l}0.186^{\mathrm{A}} \\
0.196^{\mathrm{B}}\end{array}$ & $0.184^{a}$ & $\begin{array}{l}1.91 \times 10^{7 \mathrm{~A}} \\
2.04 \times 10^{7 \mathrm{~B}}\end{array}$ & $\begin{array}{l}1.91 \times 10^{7 a} \\
1.92 \times 10^{7 c} \\
1.97 \times 10^{7 d} \\
1.99 \times 10^{7 e}\end{array}$ \\
\hline $4 f^{14} 6 d^{2} D_{5 / 2}$ & $4 f^{14} 5 f^{2} F_{7 / 2}^{\circ}$ & $\begin{array}{l}7899.18^{\mathrm{A}} \\
7938.71^{\mathrm{B}}\end{array}$ & $\begin{array}{l}7936.53^{a} \\
7936.447^{b}\end{array}$ & $\begin{array}{l}3.750^{\mathrm{A}} \\
3.954^{\mathrm{B}}\end{array}$ & $3.711^{a}$ & $\begin{array}{l}4.01 \times 10^{8 \mathrm{~A}} \\
4.18 \times 10^{8 \mathrm{~B}}\end{array}$ & $\begin{array}{l}3.93 \times 10^{8 a} \\
3.98 \times 10^{8 c} \\
4.06 \times 10^{8 d} \\
4.11 \times 10^{8 e}\end{array}$ \\
\hline $4 f^{14} 6 d^{2} D_{3 / 2}$ & $4 f^{14} 6 f^{2} F_{5 / 2}^{\circ}$ & $\begin{array}{l}2732.11^{\mathrm{A}} \\
2722.45^{\mathrm{B}}\end{array}$ & $2721.65^{a}$ & $\begin{array}{l}0.660^{\mathrm{A}} \\
0.744^{\mathrm{B}}\end{array}$ & $0.706^{a}$ & $\begin{array}{l}5.89 \times 10^{8 \mathrm{~A}} \\
6.69 \times 10^{8 \mathrm{~B}}\end{array}$ & $\begin{array}{l}6.30 \times 10^{8 a} \\
5.65 \times 10^{8 c} \\
5.46 \times 10^{8 d} \\
5.33 \times 10^{8 e}\end{array}$ \\
\hline
\end{tabular}


TABLE I (cont.)

\begin{tabular}{|c|c|c|c|c|c|c|c|}
\hline \multicolumn{2}{|c|}{ Transition } & \multicolumn{2}{|c|}{$\lambda[\AA]$} & \multicolumn{2}{|c|}{$g f$} & \multicolumn{2}{|c|}{$g A_{k i}\left[\mathrm{~s}^{-1}\right]$} \\
\hline Lower level & Upper level & This work & Others & This w. & Others & This work & Others \\
\hline $4 f^{14} 6 d^{2} D_{5 / 2}$ & $4 f^{14} 6 f^{2} F_{5 / 2}^{\circ}$ & $\begin{array}{l}2792.07^{\mathrm{A}} \\
2781.98^{\mathrm{B}}\end{array}$ & $\begin{array}{l}2781.16^{a} \\
2781.156^{b}\end{array}$ & $\begin{array}{l}0.046^{\mathrm{A}} \\
0.052^{\mathrm{B}}\end{array}$ & $0.049^{a}$ & $\begin{array}{l}3.95 \times 10^{7 \mathrm{~A}} \\
4.48 \times 10^{7 \mathrm{~B}}\end{array}$ & $\begin{array}{l}4.22 \times 10^{7 a, e} \\
4.46 \times 10^{7 c} \\
4.31 \times 10^{7 d}\end{array}$ \\
\hline $4 f^{14} 6 d^{2} D_{5 / 2}$ & $4 f^{14} 6 f^{2} F_{7 / 2}^{\circ}$ & $\begin{array}{l}2791.58^{\mathrm{A}} \\
2801.73^{\mathrm{B}}\end{array}$ & $\begin{array}{l}2800.91^{a} \\
2800.905^{b}\end{array}$ & $\begin{array}{l}0.921^{\mathrm{A}} \\
1.033^{\mathrm{B}}\end{array}$ & $0.987^{a}$ & $\begin{array}{l}7.89 \times 10^{8 \mathrm{~A}} \\
8.77 \times 10^{8 \mathrm{~B}}\end{array}$ & $\begin{array}{l}8.43 \times 10^{8 a} \\
8.64 \times 10^{8 c} \\
8.40 \times 10^{8 d} \\
8.24 \times 10^{8 e}\end{array}$ \\
\hline $4 f^{14} 6 d^{2} D_{3 / 2}$ & $4 f^{14} 7 f^{2} F_{5 / 2}^{\circ}$ & $2051.37^{\mathrm{A}, \mathrm{B}}$ & $\begin{array}{l}2050.71^{a} \\
2050.717^{b}\end{array}$ & $\begin{array}{l}0.326^{\mathrm{A}} \\
0.343^{\mathrm{B}}\end{array}$ & $0.323^{a}$ & $\begin{array}{l}5.17 \times 10^{8 \mathrm{~A}} \\
5.44 \times 10^{8 \mathrm{~B}}\end{array}$ & $\begin{array}{l}5.11 \times 10^{8 a} \\
5.05 \times 10^{8 c} \\
4.85 \times 10^{8 d} \\
4.84 \times 10^{8 e}\end{array}$ \\
\hline $4 f^{14} 6 d^{2} D_{5 / 2}$ & $4 f^{14} 7 f^{2} F_{5 / 2}^{\circ}$ & $2084.99^{\mathrm{A}, \mathrm{B}}$ & $2084.32^{a}$ & $\begin{array}{l}0.023^{\mathrm{A}} \\
0.024^{\mathrm{B}}\end{array}$ & $0.023^{a}$ & $\begin{array}{l}3.52 \times 10^{7 \mathrm{~A}} \\
3.70 \times 10^{7 \mathrm{~B}}\end{array}$ & $\begin{array}{l}3.48 \times 10^{7 a} \\
3.82 \times 10^{7 c} \\
3.65 \times 10^{8 d} \\
3.60 \times 10^{7 e}\end{array}$ \\
\hline $4 f^{14} 6 d^{2} D_{5 / 2}$ & $4 f^{14} 7 f^{2} F_{7 / 2}^{\circ}$ & $2084.00^{\mathrm{A}, \mathrm{B}}$ & $\begin{array}{l}2083.34^{a} \\
2083.342^{b}\end{array}$ & $\begin{array}{l}0.459^{\mathrm{A}} \\
0.483^{\mathrm{B}}\end{array}$ & $0.454^{a}$ & $\begin{array}{l}7.05 \times 10^{8 \mathrm{~A}} \\
7.42 \times 10^{8 \mathrm{~B}}\end{array}$ & $\begin{array}{l}6.97 \times 10^{8 a} \\
7.48 \times 10^{8 c} \\
7.16 \times 10^{8 d} \\
7.07 \times 10^{8 e}\end{array}$ \\
\hline $4 f^{14} 6 d^{2} D_{3 / 2}$ & $4 f^{14} 8 f^{2} F_{5 / 2}^{\circ}$ & $\begin{array}{l}1779.61^{\mathrm{A}} \\
1779.60^{\mathrm{B}}\end{array}$ & $1779.61^{a}$ & $\begin{array}{l}0.177^{\mathrm{A}} \\
0.183^{\mathrm{B}}\end{array}$ & $0.169^{a}$ & $\begin{array}{l}3.72 \times 10^{8 \mathrm{~A}} \\
3.85 \times 10^{8 \mathrm{~B}}\end{array}$ & $\begin{array}{l}3.56 \times 10^{8 a} \\
3.60 \times 10^{8 c} \\
3.44 \times 10^{8 d} \\
3.37 \times 10^{8 e}\end{array}$ \\
\hline $4 f^{14} 6 d^{2} D_{5 / 2}$ & $4 f^{14} 8 f^{2} F_{5 / 2}^{\circ}$ & $\begin{array}{l}1804.86^{\mathrm{A}} \\
1804.85^{\mathrm{B}}\end{array}$ & $1804.85^{a}$ & $\begin{array}{l}0.012^{\mathrm{A}} \\
0.013^{\mathrm{B}}\end{array}$ & $0.012^{a}$ & $\begin{array}{l}2.55 \times 10^{7 \mathrm{~A}} \\
2.64 \times 10^{7 \mathrm{~B}}\end{array}$ & $\begin{array}{l}2.44 \times 10^{7 a} \\
2.69 \times 10^{7 c} \\
2.56 \times 10^{7 d} \\
2.50 \times 10^{7 e}\end{array}$ \\
\hline $4 f^{14} 6 d^{2} D_{5 / 2}$ & $4 f^{14} 8 f^{2} F_{7 / 2}^{\circ}$ & $\begin{array}{l}1804.32^{\mathrm{A}} \\
1804.33^{\mathrm{B}}\end{array}$ & $1804.33^{a}$ & $\begin{array}{l}0.250^{\mathrm{A}} \\
0.258^{\mathrm{B}}\end{array}$ & $0.238^{a}$ & $\begin{array}{l}5.11 \times 10^{8 \mathrm{~A}} \\
5.28 \times 10^{8 \mathrm{~B}}\end{array}$ & $\begin{array}{l}4.88 \times 10^{8 a} \\
5.28 \times 10^{8 c} \\
5.05 \times 10^{8 d} \\
4.94 \times 10^{8 e}\end{array}$ \\
\hline $4 f^{14} 6 d^{2} D_{3 / 2}$ & $4 f^{14} 9 f^{2} F_{5 / 2}^{\circ}$ & $1638.75^{\mathrm{A}, \mathrm{B}}$ & $1638.75^{a}$ & $\begin{array}{l}0.103^{\mathrm{A}} \\
0.110^{\mathrm{B}}\end{array}$ & $0.100^{a}$ & $\begin{array}{l}2.56 \times 10^{8 \mathrm{~A}} \\
2.72 \times 10^{8 \mathrm{~B}}\end{array}$ & $\begin{array}{l}2.49 \times 10^{8 a} \\
2.56 \times 10^{8 c} \\
2.43 \times 10^{8 d} \\
2.35 \times 10^{8 e}\end{array}$ \\
\hline $4 f^{14} 6 d^{2} D_{5 / 2}$ & $4 f^{14} 9 f^{2} F_{5 / 2}^{\circ}$ & $1660.14^{\mathrm{A}, \mathrm{B}}$ & $1660.14^{a}$ & $\begin{array}{l}0.008^{\mathrm{A}} \\
0.007^{\mathrm{B}}\end{array}$ & $0.007^{a}$ & $\begin{array}{l}1.76 \times 10^{7 \mathrm{~A}} \\
1.87 \times 10^{7 \mathrm{~B}}\end{array}$ & $\begin{array}{l}1.71 \times 10^{7 a} \\
1.90 \times 10^{7 c} \\
1.80 \times 10^{7 d} \\
1.75 \times 10^{7 e}\end{array}$ \\
\hline $4 f^{14} 6 d^{2} D_{5 / 2}$ & $4 f^{14} 9 f^{2} F_{7 / 2}^{\circ}$ & $1659.84^{\mathrm{A}, \mathrm{B}}$ & $1659.84^{a}$ & $\begin{array}{l}0.146^{\mathrm{A}} \\
0.154^{\mathrm{B}}\end{array}$ & $0.141^{a}$ & $\begin{array}{l}3.53 \times 10^{8 \mathrm{~A}} \\
3.74 \times 10^{8 \mathrm{~B}}\end{array}$ & $\begin{array}{l}3.42 \times 10^{8 a} \\
3.74 \times 10^{8 c} \\
3.55 \times 10^{8 d} \\
3.46 \times 10^{8 e}\end{array}$ \\
\hline $4 f^{14} 5 f^{2} F_{5 / 2}^{\circ}$ & $4 f^{14} 7 d^{2} D_{3 / 2}$ & $\begin{array}{l}5850.02^{\mathrm{A}} \\
5871.37^{\mathrm{B}}\end{array}$ & $\begin{array}{l}5869.71^{a} \\
5869.712^{b}\end{array}$ & $\begin{array}{l}0.564^{\mathrm{A}} \\
0.476^{\mathrm{B}}\end{array}$ & $0.485^{a}$ & $\begin{array}{l}10.98 \times 10^{7 \mathrm{~A}} \\
9.20 \times 10^{7 \mathrm{~B}}\end{array}$ & $9.38 \times 10^{7 a}$ \\
\hline $4 f^{14} 5 f^{2} F_{5 / 2}^{\circ}$ & $4 f^{14} 7 d^{2} D_{5 / 2}$ & $\begin{array}{l}5729.85^{\mathrm{A}} \\
5750.33^{\mathrm{B}}\end{array}$ & $\begin{array}{l}5748.71^{a} \\
5748.712^{b}\end{array}$ & $\begin{array}{l}0.040^{\mathrm{A}} \\
0.035^{\mathrm{B}}\end{array}$ & $0.035^{a}$ & $\begin{array}{l}8.19 \times 10^{6 \mathrm{~A}} \\
7.00 \times 10^{6 \mathrm{~B}}\end{array}$ & $7.13 \times 10^{6 a}$ \\
\hline $4 f^{14} 5 f^{2} F_{7 / 2}^{\circ}$ & $4 f^{14} 7 d^{2} D_{5 / 2}$ & $\begin{array}{l}5809.29^{\mathrm{A}} \\
5788.08^{\mathrm{B}}\end{array}$ & $\begin{array}{l}5786.47^{a} \\
5786.460^{b}\end{array}$ & $\begin{array}{l}0.840^{\mathrm{A}} \\
0.689^{\mathrm{B}}\end{array}$ & $0.703^{a}$ & $\begin{array}{l}1.66 \times 10^{8 \mathrm{~A}} \\
1.37 \times 10^{8 \mathrm{~B}}\end{array}$ & $1.40 \times 10^{8 a}$ \\
\hline $4 f^{14} 7 d^{2} D_{3 / 2}$ & $4 f^{14} 7 f^{2} F_{5 / 2}^{\circ}$ & $\begin{array}{l}5420.93^{\mathrm{A}} \\
5420.94^{\mathrm{B}}\end{array}$ & $\begin{array}{l}5419.40^{a} \\
5419.418^{b}\end{array}$ & $\begin{array}{l}0.291^{\mathrm{A}} \\
0.473^{\mathrm{B}}\end{array}$ & $0.459^{a}$ & $\begin{array}{l}6.60 \times 10^{7 \mathrm{~A}} \\
1.07 \times 10^{8 \mathrm{~B}}\end{array}$ & $1.04 \times 10^{8 a}$ \\
\hline $4 f^{14} 7 d^{2} D_{5 / 2}$ & $4 f^{14} 7 f^{2} F_{5 / 2}^{\circ}$ & $5528.37^{\mathrm{A}, \mathrm{B}}$ & $\begin{array}{l}5526.81^{a} \\
5526.799^{b}\end{array}$ & $\begin{array}{l}0.025^{\mathrm{A}} \\
0.033^{\mathrm{B}}\end{array}$ & $0.032^{a}$ & $\begin{array}{l}5.54 \times 10^{6 \mathrm{~A}} \\
7.23 \times 10^{6 \mathrm{~B}}\end{array}$ & $7.01 \times 10^{6 a}$ \\
\hline
\end{tabular}


TABLE I (cont.)

\begin{tabular}{|c|c|c|c|c|c|c|c|}
\hline \multicolumn{2}{|c|}{ Transition } & \multicolumn{2}{|c|}{$\lambda[\AA]$} & \multicolumn{2}{|c|}{$g f$} & \multicolumn{2}{|c|}{$g A_{k i}\left[\mathrm{~s}^{-1}\right]$} \\
\hline Lower level & Upper level & This work & Others & This w. & Others & This work & Others \\
\hline $4 f^{14} 7 d^{2} D_{5 / 2}$ & $4 f^{14} 7 f^{2} F_{7 / 2}^{\circ}$ & $\begin{array}{l}5521.45^{\mathrm{A}} \\
5521.43^{\mathrm{B}}\end{array}$ & $\begin{array}{l}5519.91^{a} \\
5519.877^{b}\end{array}$ & $\begin{array}{l}0.507^{\mathrm{A}} \\
0.663^{\mathrm{B}}\end{array}$ & $0.643^{a}$ & $\begin{array}{l}1.11 \times 10^{8 \mathrm{~A}} \\
1.45 \times 10^{8 \mathrm{~B}}\end{array}$ & $1.41 \times 10^{8 a}$ \\
\hline $4 f^{14} 7 d^{2} D_{3 / 2}$ & $4 f^{14} 8 f^{2} F_{5 / 2}^{\circ}$ & $\begin{array}{l}3862.32^{\mathrm{A}} \\
3862.29^{\mathrm{B}}\end{array}$ & $3861.21^{a}$ & $\begin{array}{l}0.173^{\mathrm{A}} \\
0.249^{\mathrm{B}}\end{array}$ & $0.244^{a}$ & $\begin{array}{l}0.78 \times 10^{8 \mathrm{~A}} \\
1.11 \times 10^{8 \mathrm{~B}}\end{array}$ & $1.09 \times 10^{8 a}$ \\
\hline $4 f^{14} 7 d^{2} D_{5 / 2}$ & $4 f^{14} 8 f^{2} F_{5 / 2}^{\circ}$ & $\begin{array}{l}3916.55^{\mathrm{A}} \\
3916.52^{\mathrm{B}}\end{array}$ & $3915.42^{a}$ & $\begin{array}{l}0.015^{\mathrm{A}} \\
0.018^{\mathrm{B}}\end{array}$ & $0.017^{a}$ & $\begin{array}{l}6.37 \times 10^{6 \mathrm{~A}} \\
7.63 \times 10^{6 \mathrm{~B}}\end{array}$ & $7.46 \times 10^{6 a}$ \\
\hline $4 f^{14} 7 d^{2} D_{5 / 2}$ & $4 f^{14} 8 f^{2} F_{7 / 2}^{\circ}$ & $\begin{array}{l}3914.05^{\mathrm{B}} \\
3914.04^{\mathrm{A}}\end{array}$ & $3912.94^{a}$ & $\begin{array}{l}0.293^{\mathrm{A}} \\
0.351^{\mathrm{B}}\end{array}$ & $0.343^{a}$ & $\begin{array}{l}1.28 \times 10^{8 \mathrm{~A}} \\
1.53 \times 10^{8 \mathrm{~B}}\end{array}$ & $1.50 \times 10^{8 a}$ \\
\hline $4 f^{14} 7 d^{2} D_{3 / 2}$ & $4 f^{14} 9 f^{2} F_{5 / 2}^{\circ}$ & $3255.09^{\mathrm{A}, \mathrm{B}}$ & $3254.15^{a}$ & $\begin{array}{l}0.084^{\mathrm{A}} \\
0.141^{\mathrm{B}}\end{array}$ & $0.138^{a}$ & $\begin{array}{l}5.27 \times 10^{7 \mathrm{~A}} \\
8.86 \times 10^{7 \mathrm{~B}}\end{array}$ & $8.66 \times 10^{7 a}$ \\
\hline $4 f^{14} 7 d^{2} D_{5 / 2}$ & $4 f^{14} 9 f^{2} F_{5 / 2}^{\circ}$ & $\begin{array}{l}3293.52^{\mathrm{A}} \\
3293.53^{\mathrm{B}}\end{array}$ & $3292.58^{a}$ & $\begin{array}{l}0.008^{\mathrm{A}} \\
0.010^{\mathrm{B}}\end{array}$ & $0.010^{a}$ & $\begin{array}{l}4.82 \times 10^{6 \mathrm{~A}} \\
6.11 \times 10^{6 \mathrm{~B}}\end{array}$ & $5.97 \times 10^{6 a}$ \\
\hline $4 f^{14} 7 d^{2} D_{3 / 2}$ & $4 f^{14} 9 f^{2} F_{7 / 2}^{\circ}$ & $3292.35^{\mathrm{A}, \mathrm{B}}$ & $3291.40^{a}$ & $\begin{array}{l}0.157^{\mathrm{A}} \\
0.199^{\mathrm{B}}\end{array}$ & $0.194^{a}$ & $\begin{array}{l}0.96 \times 10^{8 \mathrm{~A}} \\
1.22 \times 10^{8 \mathrm{~B}}\end{array}$ & $1.20 \times 10^{8 a}$ \\
\hline $4 f^{14} 5 f^{2} F_{5 / 2}^{\circ}$ & $4 f^{14} 5 g^{2} G_{7 / 2}$ & $\begin{array}{l}4241.43^{\mathrm{A}} \\
4252.66^{\mathrm{B}}\end{array}$ & $\begin{array}{l}4251.44^{a} \\
4251.441^{b} \\
4217.750^{h}\end{array}$ & $\begin{array}{l}7.727^{\mathrm{A}} \\
7.753^{\mathrm{B}}\end{array}$ & $7.253^{a}$ & $\begin{array}{l}2.87 \times 10^{9 \mathrm{~A}} \\
2.86 \times 10^{9 \mathrm{~B}}\end{array}$ & $2.68 \times 10^{9 a}$ \\
\hline $4 f^{14} 5 f^{2} F_{7 / 2}^{\circ}$ & $4 f^{14} 5 g^{2} G_{7 / 2}$ & $\begin{array}{l}4284.80^{\mathrm{A}} \\
4273.27^{\mathrm{B}}\end{array}$ & $\begin{array}{l}4272.07^{a} \\
4238.125^{h}\end{array}$ & $\begin{array}{l}0.284^{\mathrm{A}} \\
0.286^{\mathrm{B}}\end{array}$ & $0.267^{a}$ & $\begin{array}{l}10.31 \times 10^{7 \mathrm{~A}} \\
10.44 \times 10^{7 \mathrm{~B}}\end{array}$ & $9.77 \times 10^{7 a}$ \\
\hline $4 f^{14} 5 f^{2} F_{7 / 2}^{\circ}$ & $4 f^{14} 5 g^{2} G_{9 / 2}$ & $\begin{array}{l}4284.66^{\mathrm{A}} \\
4273.10^{\mathrm{B}}\end{array}$ & $\begin{array}{l}4271.90^{a} \\
4271.908^{b} \\
4238.152^{h}\end{array}$ & $\begin{array}{l}9.926^{\mathrm{A}} \\
10.002^{\mathrm{B}}\end{array}$ & $9.358^{a}$ & $\begin{array}{l}3.61 \times 10^{9 \mathrm{~A}} \\
3.65 \times 10^{9 \mathrm{~B}}\end{array}$ & $3.42 \times 10^{9 a}$ \\
\hline $4 f^{14} 5 g^{2} G_{7 / 2}$ & $4 f^{14} 7 f^{2} F_{5 / 2}^{\circ}$ & $\begin{array}{l}8358.38^{\mathrm{A}} \\
8358.34^{\mathrm{B}}\end{array}$ & $8355.97^{a}$ & $\begin{array}{l}0.080^{\mathrm{A}} \\
0.090^{\mathrm{B}}\end{array}$ & $0.101^{a}$ & $\begin{array}{l}7.68 \times 10^{6 \mathrm{~A}} \\
8.57 \times 10^{6 \mathrm{~B}}\end{array}$ & $9.60 \times 10^{6 a}$ \\
\hline $4 f^{14} 5 g^{2} G_{7 / 2}$ & $4 f^{14} 7 f^{2} F_{7 / 2}^{\circ}$ & $\begin{array}{l}8342.58^{\mathrm{A}} \\
8342.48^{\mathrm{B}}\end{array}$ & $8340.22^{a}$ & $0.003^{\mathrm{A}, \mathrm{B}}$ & $0.004^{a}$ & $\begin{array}{l}2.84 \times 10^{5 \mathrm{~A}} \\
3.19 \times 10^{5 \mathrm{~B}}\end{array}$ & $3.57 \times 10^{5 a}$ \\
\hline $4 f^{14} 5 g^{2} G_{9 / 2}$ & $4 f^{14} 7 f^{2} F_{7 / 2}^{\circ}$ & $\begin{array}{l}8343.12^{\mathrm{A}} \\
8343.10^{\mathrm{B}}\end{array}$ & $8340.84^{a}$ & $\begin{array}{l}0.103^{\mathrm{A}} \\
0.117^{\mathrm{B}}\end{array}$ & $0.131^{a}$ & $\begin{array}{l}1.12 \times 10^{7 \mathrm{~A}} \\
0.99 \times 10^{7 \mathrm{~B}}\end{array}$ & $1.25 \times 10^{7 a}$ \\
\hline $4 f^{14} 5 g^{2} G_{7 / 2}$ & $4 f^{14} 8 f^{2} F_{5 / 2}^{\circ}$ & $\begin{array}{l}5152.46^{\mathrm{A}} \\
5152.38^{\mathrm{B}}\end{array}$ & $5150.97^{a}$ & $\begin{array}{l}0.013^{\mathrm{A}} \\
0.014^{\mathrm{B}}\end{array}$ & $0.015^{a}$ & $\begin{array}{l}3.17 \times 10^{6 \mathrm{~A}} \\
3.44 \times 10^{6 \mathrm{~B}}\end{array}$ & $3.78 \times 10^{6 a}$ \\
\hline $4 f^{14} 5 g^{2} G_{9 / 2}$ & $4 f^{14} 8 f^{2} F_{7 / 2}^{\circ}$ & $\begin{array}{l}5148.35^{\mathrm{A}} \\
5148.33^{\mathrm{B}}\end{array}$ & $5146.92^{a}$ & $\begin{array}{l}0.016^{\mathrm{A}} \\
0.018^{\mathrm{B}}\end{array}$ & $0.020^{a}$ & $\begin{array}{l}4.06 \times 10^{6 \mathrm{~A}} \\
4.47 \times 10^{6 \mathrm{~B}}\end{array}$ & $4.91 \times 10^{6 a}$ \\
\hline $4 f^{14} 5 g^{2} G_{7 / 2}$ & $4 f^{14} 9 f^{2} F_{5 / 2}^{\circ}$ & $\begin{array}{l}4125.73^{\mathrm{A}} \\
4125.72^{\mathrm{B}}\end{array}$ & $4124.56^{a}$ & $\begin{array}{l}0.004^{\mathrm{A}} \\
0.005^{\mathrm{B}}\end{array}$ & $0.005^{a}$ & $\begin{array}{l}1.65 \times 10^{6 \mathrm{~A}} \\
1.76 \times 10^{6 \mathrm{~B}}\end{array}$ & $1.92 \times 10^{6 a}$ \\
\hline $4 f^{14} 5 g^{2} G_{9 / 2}$ & $4 f^{14} 9 f^{2} F_{7 / 2}^{\circ}$ & $\begin{array}{l}4124.02^{\mathrm{A}} \\
4124.03^{\mathrm{B}}\end{array}$ & $4122.85^{a}$ & $\begin{array}{l}0.005^{\mathrm{A}} \\
0.006^{\mathrm{B}}\end{array}$ & $0.006^{a}$ & $\begin{array}{l}2.10 \times 10^{6 \mathrm{~A}} \\
2.29 \times 10^{6 \mathrm{~B}}\end{array}$ & $2.50 \times 10^{6 a}$ \\
\hline
\end{tabular}

${ }^{a}$ Ref. [11]

${ }^{b}$ Ref. [1]

${ }^{c}$ Ref. [8], relativistic model potential approach (RMP, converted from $A_{k i}$ )

${ }^{d}$ Ref. [8], relativistic model potential approach with core-polarization effects included using $\alpha_{d}=5.20 a_{0}^{3}$ (RMP-CP Ia, converted from $A_{k i}$ )

${ }^{e}$ Ref. [8], relativistic model potential approach with core-polarization effects included using $\alpha_{d}=8.20 a_{0}^{3}$ (RMP-CP Ib, converted from $A_{k i}$ )

${ }^{f}$ Ref. [7], relativistic model potential results without core polarization (converted from $f$ ) ${ }^{g 1, g 2}$ Ref. [7], relativistic Hartree-Fock results with allowance for core polarization (g1) in potential only and (g2) in both potential and transition operator (converted from $f$ )

${ }^{h}$ Ref. [6] 
New $\lambda, g f$, and $g A_{k i}$ for electric dipole (E1) transitions in Lu III.

TABLE II

\begin{tabular}{|c|c|c|c|c|}
\hline \multicolumn{2}{|c|}{ Transition } & $\lambda[\AA]$ & $g f$ & $g A_{k i}\left[\mathrm{~s}^{-1}\right]$ \\
\hline Lower level & level & \multicolumn{3}{|c|}{ This work } \\
\hline \multirow{2}{*}{$4 f^{14} 5 d^{2} D_{3 / 2}$} & \multirow[t]{2}{*}{$4 f^{14} 8 p^{2} P_{3 / 2}^{\circ}$} & $825.98^{\mathrm{A}}$ & $0.003^{\mathrm{A}}$ & $2.93 \times 10^{7 \mathrm{~A}}$ \\
\hline & & 826 & $0.002^{\mathrm{B}}$ & $2.37 \times 10^{7 \mathrm{~B}}$ \\
\hline \multirow{2}{*}{$4 f^{14} 5 d^{2} D_{3 / 2}$} & \multirow{2}{*}{$4 f^{14} 8 p^{2} P_{1 / 2}^{\circ}$} & $832.81^{\mathrm{A}}$ & $0.015^{\mathrm{A}}$ & $1.46 \times 10^{8 \mathrm{~A}}$ \\
\hline & & $833.70^{\mathrm{B}}$ & $0.012^{\mathrm{B}}$ & $1.16 \times 10^{8 \mathrm{~B}}$ \\
\hline \multirow{2}{*}{$4 f^{14} 5 d^{2} D_{5 / 2}$} & \multirow{2}{*}{$4 f^{14} 8 p^{2} P_{3 / 2}^{\circ}$} & $846.53^{\mathrm{A}}$ & $0.028^{\mathrm{A}}$ & $2.59 \times 10^{8 \mathrm{~A}}$ \\
\hline & & $847.42^{\mathrm{B}}$ & $0.021^{\mathrm{B}}$ & $1.98 \times 10^{8 \mathrm{~B}}$ \\
\hline \multirow{2}{*}{$4 f^{14} 6 p^{2} P_{1 / 2}^{\circ}$} & \multirow{2}{*}{$4 f^{14} 10 d^{2} D_{3 / 2}$} & $885.30^{\mathrm{A}}$ & $0.039^{\mathrm{A}}$ & $3.28 \times 10^{8 \mathrm{~A}}$ \\
\hline & & 881. & $0.022^{\mathrm{B}}$ & $1.89 \times 10^{8 \mathrm{~B}}$ \\
\hline \multirow[t]{2}{*}{$4 f^{14} 6 p^{2} P_{3 / 2}^{\circ}$} & \multirow[t]{2}{*}{$4 f^{14} 10 d^{2} D_{3 / 2}$} & $937.63^{\mathrm{A}}$ & $0.009^{\mathrm{A}}$ & $6.50 \times 10^{7 \mathrm{~A}}$ \\
\hline & & 932. & 0.0 & $3.19 \times 10^{7 \mathrm{~B}}$ \\
\hline \multirow[t]{2}{*}{$4 f^{14} 6 p^{2} P_{3 / 2}^{\circ}$} & \multirow[t]{2}{*}{$4 f^{14} 10 d^{2} D_{5 / 2}$} & $937.30^{\mathrm{A}}$ & $0.085^{\mathrm{A}}$ & $6.44 \times 10^{8 \mathrm{~A}}$ \\
\hline & & 932. & $0.037^{\mathrm{B}}$ & $2.88 \times$ \\
\hline $4 f^{13} 5 d\left({ }^{3} P^{\circ}\right) 6$ & $\left({ }^{3} F^{\circ}\right) 6 p^{2} D_{3 / 2}$ & $1119.40^{\mathrm{A}}$ & $0.078^{\mathrm{A}}$ & $4.14 \times 10^{8 \mathrm{~A}}$ \\
\hline $4 f^{13} 5 d^{2}\left({ }^{3} F^{\circ}\right)^{2} I_{11 / 2}^{\circ}$ & $4 f^{13} 6 s\left({ }^{3} F^{\circ}\right) 6 p^{2} G_{9 / 2}$ & $1147.96^{\mathrm{A}}$ & $0.062^{\mathrm{A}}$ & $3.14 \times 10^{8 \mathrm{~A}}$ \\
\hline $4 f^{13} 5 d\left({ }^{3} P^{\circ}\right) 6 s^{2} P_{3 / 2}^{\circ}$ & $4 f^{13} 6 s\left({ }^{3} F^{\circ}\right) 6 p^{2} D_{5 / 2}$ & $1172.23^{\mathrm{A}}$ & $0.258^{\mathrm{A}}$ & $1.25 \times 10^{9 \mathrm{~A}}$ \\
\hline $4 f^{13} 5 d\left({ }^{3} D^{\circ}\right) 6 s{ }^{4} D_{7 / 2}^{\circ}$ & $4 f^{13} 6 s\left({ }^{3} F^{\circ}\right) 6 p^{2} F_{7 / 2}$ & $1221.01^{\mathrm{A}}$ & $0.041^{\mathrm{A}}$ & $1.82 \times 10^{8 \mathrm{~A}}$ \\
\hline $4 f^{13} 5 d\left({ }^{3} P^{\circ}\right) 6$ & $4 f^{13} 6 s\left({ }^{3} F^{\circ}\right) 6 p^{2} D_{5 / 2}$ & $1257.01^{\mathrm{A}}$ & $0.168^{\mathrm{A}}$ & $7.10 \times 10^{8 \mathrm{~A}}$ \\
\hline $4 f^{13} 5 d\left({ }^{3} P^{\circ}\right) 6 s{ }^{4} P_{3 / 2}^{\circ}$ & $4 f^{13} 6 s\left({ }^{3} F^{\circ}\right) 6 p^{4} F_{5 / 2}$ & $1478.68^{\mathrm{A}}$ & $0.033^{\mathrm{A}}$ & $1.00 \times 10^{8 \mathrm{~A}}$ \\
\hline $4 f^{13} 5 d\left({ }^{3} P^{\circ}\right) 6 s^{4} P_{5 / 2}^{\circ}$ & $4 f^{13} 6 s\left({ }^{3} F^{\circ}\right) 6 p{ }^{4} F_{7 / 2}$ & $1582.91^{\mathrm{A}}$ & $0.064^{\mathrm{A}}$ & $1.71 \times 10^{8 \mathrm{~A}}$ \\
\hline $4 f^{13} 5 d\left({ }^{3} P^{\circ}\right) 6 s{ }^{4} P_{3 / 2}^{\circ}$ & $4 f^{13} 6 s\left({ }^{1} F^{\circ}\right) 6 p^{2} D_{5 / 2}$ & $1622.94^{\mathrm{A}}$ & $0.111^{\mathrm{A}}$ & $2.82 \times 10^{8 \mathrm{~A}}$ \\
\hline $4 f^{13} 5 d^{2}\left({ }^{3} F^{\circ}\right)^{4} I_{13 / 2}^{\circ}$ & $4 f^{13} 6 s\left({ }^{3} F^{\circ}\right) 6 p^{4} G_{11 / 2}$ & $1673.63^{\mathrm{A}}$ & $0.042^{\mathrm{A}}$ & $1.01 \times 10^{8 \mathrm{~A}}$ \\
\hline $4 f^{13} 5 d\left({ }^{3} P^{\circ}\right) 6 s{ }^{4} P_{5 / 2}^{\circ}$ & $4 f^{13} 5 d\left({ }^{3} G^{\circ}\right) 6 p^{2} F_{7 / 2}$ & $1690.66^{\mathrm{A}}$ & $0.073^{\mathrm{A}}$ & $1.71 \times 10^{8 \mathrm{~A}}$ \\
\hline $4 f^{13} 5 d\left({ }^{3} P^{\circ}\right) 6 s{ }^{4} P_{1 / 2}^{\circ}$ & $4 f^{13} 5 d\left({ }^{1} P^{\circ}\right) 6 p^{2} D_{3 / 2}$ & $1694.68^{\mathrm{A}}$ & $0.059^{\mathrm{A}}$ & $1.38 \times 10^{8 \mathrm{~A}}$ \\
\hline $4 f^{13} 5 d\left({ }^{3} P^{\circ}\right) 6 s^{2} P_{3 / 2}^{\circ}$ & $4 f^{13} 5 d\left({ }^{3} D^{\circ}\right) 6 p^{2} D_{5 / 2}$ & $1698.78^{\mathrm{A}}$ & $0.144^{\mathrm{A}}$ & $3.32 \times 10^{8 \mathrm{~A}}$ \\
\hline $4 f^{13} 5 d^{2}\left({ }^{3} F^{\circ}\right)^{4} I_{13 / 2}^{\circ}$ & $4 f^{13} 5 d\left({ }^{3} H^{\circ}\right) 6 p^{4} I_{11 / 2}$ & $1713.09^{\mathrm{A}}$ & $0.046^{\mathrm{A}}$ & $1.04 \times 10^{8 \mathrm{~A}}$ \\
\hline $4 f^{13} 5 d^{2}\left({ }^{3} F^{\circ}\right){ }^{4} F_{?}$ & $4 f^{13} 5 d\left({ }^{3} G^{\circ}\right) 6 p{ }^{4} G_{11 / 2}$ & $1713.63^{\mathrm{A}}$ & $0.0^{\prime}$ & $1.60 \times 10^{8 \mathrm{~A}}$ \\
\hline $4 f^{13} 5 d\left({ }^{3} P^{\circ}\right) 6 s{ }^{4} P_{5 / 2}^{\circ}$ & $4 f^{13} 5 d\left({ }^{1} G^{\circ}\right) 6 p^{2} G_{7 / 2}$ & $1737.31^{\mathrm{A}}$ & 0.14 & $3.11 \times 10^{8 \mathrm{~A}}$ \\
\hline $4 f^{13} 5 d^{2}\left({ }^{3} F^{\circ}\right){ }^{4} F_{9}^{\circ}$ & $4 f^{13} 6 s\left({ }^{3} F^{\circ}\right) 6 p^{4} D_{7 / 2}$ & $1738.40^{\mathrm{A}}$ & 0.0 & $1.06 \times 10^{8 \mathrm{~A}}$ \\
\hline $4 f^{13} 5 d\left({ }^{3} P^{\circ}\right) 6 s^{2} P_{3 / 2}^{\circ}$ & $4 f^{13} 5 d\left({ }^{3} H^{\circ}\right) 6 p{ }^{4} G_{5 / 2}$ & $1745.47^{\mathrm{A}}$ & $0.196^{\mathrm{A}}$ & $4.28 \times 10^{8 \mathrm{~A}}$ \\
\hline $4 f^{13} 5 d\left({ }^{3} P^{\circ}\right) 6 s{ }^{4} P_{5 / 2}^{\circ}$ & $4 f^{13} 6 s\left({ }^{3} F^{\circ}\right) 6 p^{4} D_{7 / 2}$ & $1750.49^{\mathrm{A}}$ & 1.0 & $2.32 \times 10^{9 \mathrm{~A}}$ \\
\hline $4 f^{13} 5 d\left({ }^{3} D^{\circ}\right) 6 s{ }^{4} D_{7 / 2}^{\circ}$ & $4 f^{13} 6 s\left({ }^{3} G^{\circ}\right) 6 p^{2} G_{9 / 2}$ & $1754.73^{\mathrm{A}}$ & $0.086^{\mathrm{A}}$ & $1.85 \times 10^{8 \mathrm{~A}}$ \\
\hline $4 f^{13} 5 d\left({ }^{3} P^{\circ}\right) 6 s{ }^{4} P_{3 / 2}^{\circ}$ & $4 f^{13} 5 d\left({ }^{3} D^{\circ}\right) 6 p^{4} F_{5 / 2}$ & $1759.35^{\mathrm{A}}$ & $0.052^{\mathrm{A}}$ & $1.13 \times 10^{8 \mathrm{~A}}$ \\
\hline $4 f^{13} 5 d\left({ }^{3} D^{\circ}\right) 6 s^{4} D_{7 / 2}^{\circ}$ & $4 f^{13} 6 s\left({ }^{3} F^{\circ}\right) 6 p^{4} F_{9 / 2}$ & 1769 & $0.344^{\mathrm{A}}$ & $7.33 \times 10^{8 \mathrm{~A}}$ \\
\hline $4 f^{13} 5 d\left({ }^{3} P^{\circ}\right) 6 s{ }^{4} P_{1 / 2}^{\circ}$ & $4 f^{13} 6 s\left({ }^{3} F^{\circ}\right) 6 p^{4} D_{3 / 2}$ & $1775.92^{\mathrm{A}}$ & $0.069^{\mathrm{A}}$ & $1.47 \times 10^{8 \mathrm{~A}}$ \\
\hline $4 f^{13} 5 d^{2}\left({ }^{3} F^{\circ}\right){ }^{4} I_{13 / 2}^{\circ}$ & $4 f^{13} 5 d\left({ }^{3} G^{\circ}\right) 6 p^{4} G_{11 / 2}$ & 1777.9 & $0.060^{\mathrm{A}}$ & $1.27 \times 10^{8 \mathrm{~A}}$ \\
\hline $4 f^{13} 5 d\left({ }^{3} D^{\circ}\right) 6 s^{4} D_{5 / 2}^{\circ}$ & $4 f^{13} 6 s\left({ }^{3} F^{\circ}\right) 6 p^{4} F_{7 / 2}$ & $1810.20^{\mathrm{A}}$ & $0.226^{\mathrm{A}}$ & $4.60 \times 10^{8 \mathrm{~A}}$ \\
\hline $4 f^{13} 5 d^{2}\left({ }^{3} F^{\circ}\right){ }^{4} P_{3 / 2}^{\circ}$ & $4 f^{13} 5 d\left({ }^{3} F^{\circ}\right) 6 p^{2} D_{5 / 2}$ & $1811 . ?$ & $0.093^{\mathrm{A}}$ & $1.89 \times 10^{8 \mathrm{~A}}$ \\
\hline $4 f^{13} 5 d^{2}\left({ }^{3} F^{\circ}\right)^{2} I_{11 / 2}^{\circ}$ & $4 f^{13} 5 d\left({ }^{3} H^{\circ}\right) 6 p^{2} G_{9 / 2}$ & $1811.89^{\mathrm{A}}$ & $0.061^{\mathrm{A}}$ & $1.24 \times 10^{8 \mathrm{~A}}$ \\
\hline $4 f^{13} 5 d^{2}\left({ }^{3} F^{\circ}\right){ }^{4} F_{7 / 2}^{\circ}$ & $4 f^{13} 5 d\left({ }^{3} F^{\circ}\right) 6 p^{4} F_{9 / 2}$ & 1819. & $0.228^{\mathrm{A}}$ & $4.58 \times 10^{8 \mathrm{~A}}$ \\
\hline $4 f^{13} 5 d^{2}\left({ }^{3} F^{\circ}\right){ }^{4} P_{1}^{\circ}$ & $4 f^{13} 5 d\left({ }^{3} P^{\circ}\right) 6 p^{2} D_{3 / 2}$ & 1820.9 & $0.052^{\mathrm{A}}$ & $1.04 \times 10^{8 \mathrm{~A}}$ \\
\hline $4 f^{13} 5 d\left({ }^{3} D^{\circ}\right) 6 s{ }^{4} D_{7 / 2}^{\circ}$ & $4 f^{13} 5 d\left({ }^{3} H^{\circ}\right) 6 p{ }^{4} H_{9 / 2}$ & $1826.62^{\mathrm{A}}$ & $0.089^{\mathrm{A}}$ & $1.78 \times 10^{8 \mathrm{~A}}$ \\
\hline $4 f^{13} 5 d\left({ }^{3} D^{\circ}\right) 6 s{ }^{4} D_{5 / 2}^{\circ}$ & $4 f^{13} 5 d\left({ }^{3} F^{\circ}\right) 6 p^{2} F_{7 / 2}$ & $1835.99^{\mathrm{A}}$ & $0.167^{\mathrm{A}}$ & $3.30 \times 10^{8 \mathrm{~A}}$ \\
\hline $4 f^{13} 5 d\left({ }^{3} P^{\circ}\right) 6 s^{2} P_{3 / 2}^{\circ}$ & $4 f^{13} 5 d\left({ }^{1} F^{\circ}\right) 6 p^{2} D_{5 / 2}$ & $1849.68^{\mathrm{A}}$ & $0.068^{\mathrm{A}}$ & $1.33 \times 10^{8 \mathrm{~A}}$ \\
\hline $4 f^{13} 5 d\left({ }^{3} D^{\circ}\right) 6 s{ }^{4} D_{5 / 2}^{\circ}$ & $4 f^{13} 5 d\left({ }^{3} H^{\circ}\right) 6 p{ }^{4} H_{7 / 2}$ & $1859.84^{\mathrm{A}}$ & $0.052^{\mathrm{A}}$ & $1.00 \times 10^{8 \mathrm{~A}}$ \\
\hline $4 f^{13} 5 d\left({ }^{3} P^{\circ}\right) 6 s{ }^{4} P_{1 / 2}^{\circ}$ & $4 f^{13} 5 d\left({ }^{3} F^{\circ}\right) 6 p^{4} F_{3 / 2}$ & $1863.12^{\mathrm{A}}$ & $0.082^{\mathrm{A}}$ & $1.58 \times 10^{8 \mathrm{~A}}$ \\
\hline $4 f^{13} 5 d\left({ }^{3} P^{\circ}\right) 6 s^{4} P_{5 / 2}^{\circ}$ & $4 f^{13} 5 d\left({ }^{1} D^{\circ}\right) 6 p^{2} F_{7 / 2}$ & $1867.12^{\mathrm{A}}$ & $0.158^{\mathrm{A}}$ & $3.02 \times 10^{8 \mathrm{~A}}$ \\
\hline $4 f^{13} 5 d\left({ }^{3} P^{\circ}\right) 6 s^{4} P_{1 / 2}^{\circ}$ & $4 f^{13} 5 d\left({ }^{3} D^{\circ}\right) 6 p^{4} P_{3 / 2}$ & $1881.28^{\mathrm{A}}$ & $0.102^{\mathrm{A}}$ & $1.93 \times 10^{8 \mathrm{~A}}$ \\
\hline $4 f^{13} 5 d\left({ }^{3} D^{\circ}\right) 6 s^{4} D_{5 / 2}^{\circ}$ & $4 f^{13} 5 d\left({ }^{3} H^{\circ}\right) 6 p^{4} G_{5 / 2}$ & $1887.38^{\mathrm{A}}$ & $0.142^{\mathrm{A}}$ & $2.66 \times 10^{8 \mathrm{~A}}$ \\
\hline $4 f^{13} 5 d^{2}\left({ }^{3} F^{\circ}\right)^{2} I_{11 / 2}^{\circ}$ & $4 f^{13} 5 d\left({ }^{3} F^{\circ}\right) 6 p^{4} F_{9 / 2}$ & $1893.67^{\mathrm{A}}$ & $0.132^{\mathrm{A}}$ & $2.45 \times 10^{8 \mathrm{~A}}$ \\
\hline $4 f^{13} 5 d^{2}\left({ }^{3} F^{\circ}\right){ }^{4} F_{9 / 2}^{\circ}$ & $4 f^{13} 5 d\left({ }^{3} G^{\circ}\right) 6 p{ }^{4} H_{11 / 2}$ & $1894.95^{\mathrm{A}}$ & $0.161^{\mathrm{A}}$ & $3.00 \times 10^{8 \mathrm{~A}}$ \\
\hline $4 f^{13} 5 d^{2}\left({ }^{3} F^{\circ}\right){ }^{4} F_{9 / 2}^{\circ}$ & $4 f^{13} 5 d\left({ }^{3} F^{\circ}\right) 6 p^{4} F_{9 / 2}$ & $1897.53^{\mathrm{A}}$ & $0.301^{\mathrm{A}}$ & $5.58 \times 10^{8 \mathrm{~A}}$ \\
\hline $4 f^{13} 5 d\left({ }^{3} D^{\circ}\right) 6 s{ }^{4} D_{5 / 2}^{\circ}$ & $4 f^{13} 5 d\left({ }^{3} D^{\circ}\right) 6 p^{2} D_{5 / 2}$ & $1898.77^{\mathrm{A}}$ & $0.183^{\mathrm{A}}$ & $3.38 \times 10^{8 \mathrm{~A}}$ \\
\hline
\end{tabular}


TABLE II (cont.)

\begin{tabular}{|c|c|c|c|c|}
\hline \multicolumn{2}{|c|}{ Transition } & $\lambda[\AA]$ & $g f$ & $g A_{k i}\left[\mathrm{~s}^{-1}\right]$ \\
\hline Lov & & \multicolumn{3}{|c|}{ This work } \\
\hline $4 f^{13} 5 d^{2}\left({ }^{3} F^{\circ}\right){ }^{4} F_{7 / 2}^{\circ}$ & $4 f^{13} 5 d\left({ }^{1} H^{\circ}\right.$ & 1905.03 & $0.087^{\mathrm{A}}$ & $1.61 \times 10^{8 \mathrm{~A}}$ \\
\hline $4 f^{13} 5 d\left({ }^{3} P^{\circ}\right) 6 s^{4} P_{5 / 2}^{\circ}$ & $4 f^{13} 5 d\left({ }^{3} F^{\circ}\right) 6 p^{4} D_{7 / 2}$ & $906.17^{\mathrm{A}}$ & $.193^{\mathrm{A}}$ & $3.54 \times 10^{8 \mathrm{~A}}$ \\
\hline $4 f^{13} 5 d\left({ }^{3} D^{\circ}\right) 6 s{ }^{4} D_{7 / 2}^{\circ}$ & $4 f^{13} 5 d\left({ }^{3} G^{\circ}\right) 6 p^{2} F_{7 / 2}$ & $1907.12^{\mathrm{A}}$ & $0.253^{\mathrm{A}}$ & $4.64 \times 10^{8 \mathrm{~A}}$ \\
\hline $4 f^{13} 5 d^{2}\left({ }^{3} F^{\circ}\right.$ & $4 f^{13} 5 d\left({ }^{3} H^{\circ}\right) 6 p{ }^{4} I_{9 / 2}$ & $1909.62^{\mathrm{A}}$ & $0.095^{\mathrm{A}}$ & $1.74 \times 10^{8 \mathrm{~A}}$ \\
\hline $4 f^{13} 5 d\left({ }^{3} D^{\circ}\right) 6 s{ }^{4} D_{7 / 2}^{\circ}$ & $4 f^{13} 6 s\left({ }^{3} F^{\circ}\right) 6 p^{4} G_{9 / 2}$ & $1914.68^{\mathrm{A}}$ & $0.192^{\mathrm{A}}$ & $3.50 \times 10^{8 \mathrm{~A}}$ \\
\hline $4 f^{13} 5 d\left({ }^{3} P^{\circ}\right)$ & $4 f^{13} 5 d\left({ }^{1} F^{\circ}\right) 6 p^{2} G_{7 / 2}$ & $1918.37^{\mathrm{A}}$ & $.229^{\mathrm{A}}$ & $4.14 \times 10^{8 \mathrm{~A}}$ \\
\hline $4 f^{13} 5 d\left({ }^{3} P^{\circ}\right)$ & $4 f^{13} 5 d\left({ }^{3} P^{\circ}\right) 6 p^{4} D_{5 / 2}$ & $1934.77^{\mathrm{A}}$ & $5^{\mathrm{A}}$ & $4.91 \times 10^{8 \mathrm{~A}}$ \\
\hline $4 f^{13} 5 d\left({ }^{3} P^{\circ}\right) 6 s{ }^{4} P_{1 / 2}^{\circ}$ & $4 f^{13} 5 d\left({ }^{3} D^{\circ}\right) 6 p{ }^{4} F_{3 / 2}$ & $1936.15^{\mathrm{A}}$ & $.096^{\mathrm{A}}$ & $1.71 \times 10^{8 \mathrm{~A}}$ \\
\hline $4 f^{14} 7 p^{2} P_{1 / 2}^{\circ}$ & $4 f^{14} 10 d^{2} D_{3 / 2}$ & $1940.30^{\mathrm{A}}$ & $0.015^{\mathrm{A}}$ & $2.57 \times 10^{7 \mathrm{~A}}$ \\
\hline & & 1960. & & $9.33 \times 10^{7 \mathrm{~B}}$ \\
\hline $4 f^{13} 5 d\left({ }^{3} P^{\circ}\right.$ & $4 f^{13} 5$ & 1940. & & $6.90 \times 10^{8 \mathrm{~A}}$ \\
\hline $4 f^{13} 5 d\left({ }^{3} P^{\circ}\right.$ & $4 f^{13} 5 d\left({ }^{3} D^{\circ}\right.$ & 1955. & A & $2.29 \times$ \\
\hline $4 f^{13} 5 d\left({ }^{3} D^{\circ}\right)$ & $4 f^{13} 5 d\left({ }^{3} H^{\circ}\right.$ & $1957.29^{\mathrm{A}}$ & $\mathrm{A}$ & $1.09 \times$ \\
\hline $4 f^{13} 5 d^{2}\left({ }^{3} F^{0}\right.$ & $4 f^{13} 5 d\left({ }^{3} H^{\circ}\right) 6 p^{4} G_{11 / 2}$ & $1961.04^{\mathrm{A}}$ & & 1.53 \\
\hline $4 f^{13} 5 d\left({ }^{3} P^{\circ}\right) 6 s^{2} P_{3 / 2}^{\circ}$ & $4 f^{13} 5 d\left({ }^{3} F^{\circ}\right) 6 p^{2} D_{5 / 2}$ & $1961.76^{\mathrm{A}}$ & $0.249^{\mathrm{A}}$ & $4.32 \times$ \\
\hline $4 f^{13} 5 d\left({ }^{3} D^{\circ}\right)$ & $4 f^{13} 5 d\left({ }^{1} F^{\circ}\right) 6 p^{2} G_{9 / 2}$ & $1965.71^{\mathrm{A}}$ & & \\
\hline $4 f^{13} 5 d^{2}\left({ }^{3} F^{\circ}\right){ }^{4} F_{7 / 2}^{\circ}$ & $4 f^{13} 5 d\left({ }^{3} F^{\circ}\right.$ & $1973.05^{\mathrm{A}}$ & $.207^{\mathrm{A}}$ & $3.55 \times 10^{8 \mathrm{~A}}$ \\
\hline $4 f^{13} 5 d^{2}\left({ }^{3} F^{\circ}\right){ }^{4} I_{13 / 2}^{\circ}$ & $4 f^{13} 5 d\left({ }^{3} G^{\circ}\right) 6 p^{4} H_{11 / 2}$ & $1973.91^{\mathrm{A}}$ & & $3.07 \times$ \\
\hline $4 f^{13} 5 d\left({ }^{3} D^{\circ}\right) 6 s^{4} D_{7 / 2}^{\circ}$ & $4 f^{13} 6 s\left({ }^{3} F^{\circ}\right) 6 p^{4} D_{7 / 2}$ & $1983.60^{\mathrm{A}}$ & 1. & $2.68 \times$ \\
\hline $4 f^{13} 5 d^{2}\left({ }^{3} F^{c}\right.$ & $4 f^{13} 5 d\left({ }^{1} H^{\circ}\right.$ & $1986.31^{\mathrm{A}}$ & & $2.54 \times$ \\
\hline $4 f^{13} 5 d\left({ }^{3} P^{\circ}\right)$ & $4 f^{13} 6 s\left({ }^{1} F^{\circ}\right) 6 p^{2} F_{5 / 2}$ & 1989.1 & 0.1 & $2.25 \times 1$ \\
\hline $4 f^{13} 5 d^{2}\left({ }^{3} F^{c}\right.$ & $4 f^{13} 5 d\left({ }^{3} H^{\circ}\right.$ & 1994.0 & $\mathrm{~A}$ & $2.06 \times 1$ \\
\hline $4 f^{13} 5 d^{2}\left({ }^{3} F^{\circ}\right){ }^{4} I_{13 / 2}^{\circ}$ & $4 f^{13} 5 d\left({ }^{3} H^{\circ}\right) 6 p{ }^{4} H_{13}$ & & & $8 \mathrm{~A}$ \\
\hline $4 f^{13} 5 d\left({ }^{3} P^{\circ}\right) 6 s^{2} P_{3 / 2}^{\circ}$ & $4 f^{13} 5 d\left({ }^{3} P^{\circ}\right) 6 p^{2} S_{1 / 2}$ & 2002 . & 0.2 & $3.33 \times$ \\
\hline $4 f^{13} 5 d\left({ }^{3} D^{\circ}\right.$ & $4 f^{13} 6$ & & & \\
\hline $4 f^{13} 5 d^{2}\left({ }^{3} F^{\circ}\right)^{2} I_{11 / 2}^{\circ}$ & $4 f^{13} 5 d\left({ }^{3} H^{\circ}\right) 6 p{ }^{4} H_{11 / 2}$ & 201 & 0.1 & $3.23 \times$ \\
\hline $4 f^{13} 5 d\left({ }^{3} D^{\circ}\right) 6 s^{4} D_{5 / 2}^{\circ}$ & $4 f^{13} 5 d\left({ }^{1} G^{\circ}\right) 6 p^{2} G_{7 / 2}$ & 201 & & $8 \mathrm{~A}$ \\
\hline $4 f^{13} 5 d^{2}\left({ }^{3} F^{\circ}\right){ }^{4} F_{9 / 2}^{\circ}$ & $4 f^{13} 5 d\left({ }^{3} H^{\circ}\right) 6 p{ }^{4} H_{11 / 2}$ & 2016. & 0.26 & $4.32 \times 10^{8 \mathrm{~A}}$ \\
\hline $4 f^{13} 5 d\left({ }^{3} P^{\circ}\right) 6 s{ }^{4} P_{1 / 2}^{\circ}$ & $4 f^{13} 5 d\left({ }^{3} D^{\circ}\right) 6 p^{2} P_{3 / 2}$ & 2020. & A & $1.77 \times$ \\
\hline $4 f^{13} 5 d\left({ }^{3} D^{\circ}\right)$ & $4 f^{13} 6 s\left({ }^{3} F^{\circ}\right) 6 p^{4} D_{7 / 2}$ & & & $7.88 \times$ \\
\hline $4 f^{13} 5 d^{2}\left({ }^{3} F^{\circ}\right){ }^{4} P_{1 / 2}^{\circ}$ & $4 f^{13} 5 d\left({ }^{3} D^{\circ}\right) 6 p^{4} P_{3 / 2}$ & 2033. & 0.5 & $8.50 \times 1$ \\
\hline $4 f^{13} 5 d^{2}\left({ }^{3} F^{\circ}\right){ }^{4} F_{7 / 2}^{\circ}$ & $4 f^{13} 5 d\left({ }^{3} P^{\circ}\right) 6 p^{4} D_{7 / 2}$ & 2048.6 & 0.3 & $5.85 \times 1$ \\
\hline $4 f^{14} 7 p^{2} P_{3 / 2}^{\circ}$ & $4 f^{14} 10 d^{2} D_{3 / 2}$ & 205 & 0.0 & $0.83 \times$ \\
\hline & & & & \\
\hline $4 f^{14} 7 p^{2} P_{3 / 2}^{\circ}$ & $4 f^{14} 10 d^{2} D_{5 / 2}$ & 205 & 0.0 & $1.13 \times 1$ \\
\hline & & & & $1.46 \times$ \\
\hline $4 f^{13}$ & $4 f^{1}$ & 206 & 0.2 & $3.22 \times$ \\
\hline $4 f^{13} 5 d\left({ }^{3} P^{\circ}\right)$ & $6 p^{2} D_{3 / 2}$ & 20 & & $8 \mathrm{~A}$ \\
\hline $4 f^{13} 5 d\left({ }^{3} D^{\circ}\right)$ & $4 f^{13} 5 d\left({ }^{3} D^{\circ}\right) 6 p{ }^{4} F_{9 / 2}$ & 2070. & 0.70 & $1.10 \times 10^{9 \mathrm{~A}}$ \\
\hline $4 f^{13} 5 d^{2}\left({ }^{3} F^{\circ}\right){ }^{4} P_{3 / 2}^{\circ}$ & $4 f^{13} 5 d\left({ }^{3} P^{\circ}\right) 6 p^{4} P_{5 / 2}$ & 2071. & $\mathrm{~A}$ & $2.55 \times$ \\
\hline $4 f^{13} 5 d^{2}\left({ }^{3} F^{\circ}\right){ }^{4} F_{9 / 2}^{\circ}$ & $4 f^{13} 5 d\left({ }^{3} H^{\circ}\right) 6 p^{4} G_{11 / 2}$ & $2071.98^{\mathrm{A}}$ & 0.5 & $8 \times 10^{8 \mathrm{~A}}$ \\
\hline $4 f^{13} 5 d\left({ }^{3} D^{\circ}\right) 6 s^{4} D_{7 / 2}^{\circ}$ & $4 f^{13} 5 d\left({ }^{3} H^{\circ}\right) 6 p^{2} G_{9 / 2}$ & 208 & 0.1 & $2.87 \times$ \\
\hline $4 f^{13} 5 d\left({ }^{3} D^{\circ}\right.$ & $p^{2} D_{3 / 2}$ & & & $1.08 \times 10^{8 \mathrm{~A}}$ \\
\hline $4 f^{13} 5 d^{2}\left({ }^{3} F^{\circ}\right)^{2} I_{11 / 2}^{\circ}$ & $4 f^{13} 5 d\left({ }^{3} H^{\circ}\right) 6 p^{4} I_{13 / 2}$ & 2091 & $0.083^{\mathrm{A}}$ & $1.26 \times 10^{8 \mathrm{~A}}$ \\
\hline $4 f^{13} 5 d\left({ }^{3} P^{\circ}\right.$ & $4 f^{13} 5 d\left({ }^{3} P\right.$ & 20 & 0.0 & $1.08 \times 10^{8 \mathrm{~A}}$ \\
\hline $4 f^{13} 5 d^{2}\left({ }^{3} F^{\circ}\right)^{2} I_{11 / 2}^{\circ}$ & $4 f^{13} 5 d\left({ }^{1} G^{\circ}\right) 6 p{ }^{4} H_{9 / 2}$ & $2102.54^{\mathrm{A}}$ & $1.923^{\mathrm{A}}$ & $2.90 \times 10^{9 \mathrm{~A}}$ \\
\hline $4 f^{13} 5 d^{2}\left({ }^{3} F^{\circ}\right)^{4}$ & $6 p{ }^{4} H_{11 / 2}$ & & & $1.52 \times 10^{9 \mathrm{~A}}$ \\
\hline $4 f^{13} 5 d^{2}\left({ }^{3} F^{\circ}\right){ }^{4} F_{7 / 2}^{\circ}$ & $4 f^{13} 5 d\left({ }^{1} G^{\circ}\right) 6 p^{2} H_{9 / 2}$ & 2133.7 & 0.8 & $1.27 \times 10^{9 \mathrm{~A}}$ \\
\hline $4 f^{13} 5 d\left({ }^{3} D^{\circ}\right)$ & $4 f^{13} 5 d\left({ }^{3} P^{\circ}\right) 6 p^{4} D_{5 / 2}$ & 214 & 0.2 & $3.67 \times$ \\
\hline & $5 d\left({ }^{3} P^{\circ}\right) 6 p^{4} D_{7 / 2}$ & 2147 & $0.947^{\mathrm{A}}$ & $1.37 \times 10^{9 \mathrm{~A}}$ \\
\hline $4 f^{13} 5 d\left({ }^{3} P^{\circ}\right) 6 s^{4} P_{1 / 2}^{\circ}$ & $4 f^{13} 5 d\left({ }^{1} D^{\circ}\right) 6 p^{2} P_{1 / 2}$ & $2152.74^{\mathrm{A}}$ & $0.119^{\mathrm{A}}$ & $1.72 \times 10^{8 \mathrm{~A}}$ \\
\hline $4 f^{13} 5 d\left({ }^{3} P^{\circ}\right) 6 s^{4}$ & $4 f^{13} 5 d\left({ }^{3} P^{\circ}\right) 6 p^{4} D_{7 / 2}$ & $2166.38^{\mathrm{A}}$ & $2.706^{\mathrm{A}}$ & $3.84 \times 10^{9 \mathrm{~A}}$ \\
\hline
\end{tabular}


TABLE II (cont.)

\begin{tabular}{|c|c|c|c|c|}
\hline \multicolumn{2}{|c|}{ Transition } & $\lambda[\AA]$ & $g f$ & $g A_{k i}\left[\mathrm{~s}^{-1}\right]$ \\
\hline Lower level & Upper level & \multicolumn{3}{|c|}{ This work } \\
\hline $4 f^{13} 5 d^{2}\left({ }^{3} F^{\circ}\right){ }^{4} I_{13 / 2}^{\circ}$ & $4 f^{13} 5 d\left({ }^{3} H^{\circ}\right) 6 p^{4} G_{11 / 2}$ & $2166.76^{\mathrm{A}}$ & $0.395^{\mathrm{A}}$ & $5.61 \times 10^{8 \mathrm{~A}}$ \\
\hline $4 f^{13} 5 d\left({ }^{3} P^{\circ}\right) 6 s{ }^{4} P_{3 / 2}^{\circ}$ & $4 f^{13} 5 d\left({ }^{3} P^{\circ}\right) 6 p^{4} D_{5 / 2}$ & $2177.11^{\mathrm{A}}$ & $1.172^{\mathrm{A}}$ & $1.65 \times 10^{9 \mathrm{~A}}$ \\
\hline $4 f^{13} 5 d^{2}\left({ }^{3} F^{\circ}\right){ }^{4} F_{9 / 2}^{\circ}$ & $4 f^{13} 5 d\left({ }^{3} D^{\circ}\right) 6 p^{2} F_{7 / 2}$ & $2179.68^{\mathrm{A}}$ & $0.474^{\mathrm{A}}$ & $6.65 \times 10^{8 \mathrm{~A}}$ \\
\hline $4 f^{14} 5 f^{2} F_{5 / 2}^{\circ}$ & $4 f^{14} 10 d^{2} D_{5 / 2}$ & $\begin{array}{l}2180.24^{\mathrm{A}} \\
2156.32^{\mathrm{B}}\end{array}$ & $0.001^{\mathrm{A}, \mathrm{B}}$ & $\begin{array}{l}7.05 \times 10^{5 \mathrm{~A}} \\
8.05 \times 10^{5 \mathrm{~B}}\end{array}$ \\
\hline $4 f^{13} 5 d\left({ }^{3} P^{\circ}\right) 6 s{ }^{4} P_{5 / 2}^{\circ}$ & $4 f^{13} 5 d\left({ }^{3} P^{\circ}\right) 6 p{ }^{4} S_{3 / 2}$ & $2181.61^{\mathrm{A}}$ & $0.129^{\mathrm{A}}$ & $1.81 \times 10^{8 \mathrm{~A}}$ \\
\hline \multirow[t]{2}{*}{$4 f^{14} 5 f^{2} F_{5 / 2}^{\circ}$} & $4 f^{14} 10 d^{2} D_{3 / 2}$ & $2182.05^{\mathrm{A}}$ & $0.010^{\mathrm{A}}$ & $1.36 \times 10^{7 \mathrm{~A}}$ \\
\hline & & $2159.61^{\mathrm{B}}$ & $0.008^{\mathrm{B}}$ & $1.12 \times 10^{7 \mathrm{~B}}$ \\
\hline $4 f^{13} 5 d\left({ }^{3} P^{\circ}\right) 6 s{ }^{4} P_{5 / 2}^{\circ}$ & $4 f^{13} 5 d\left({ }^{3} P^{\circ}\right) 6 p{ }^{4} P_{5 / 2}$ & $2184.26^{\mathrm{A}}$ & $0.617^{\mathrm{A}}$ & $8.63 \times 10^{8 \mathrm{~A}}$ \\
\hline \multirow{2}{*}{$4 f^{14} 5 f^{2} F_{7 / 2}^{\circ}$} & $4 f^{14} 10 d^{2} D_{5 / 2}$ & $2191.64^{\mathrm{A}}$ & $0.009^{\mathrm{A}}$ & $1.32 \times 10^{7 \mathrm{~A}}$ \\
\hline & & $2161.61^{\mathrm{B}}$ & $0.011^{\mathrm{B}}$ & $1.60 \times 10^{7 \mathrm{~B}}$ \\
\hline $4 f^{13} 5 d\left({ }^{3} D^{\circ}\right) 6 s^{4} D_{5 / 2}^{\circ}$ & $4 f^{13} 5 d\left({ }^{1} D^{\circ}\right) 6 p^{2} F_{7 / 2}$ & $2191.74^{\mathrm{A}}$ & $1.355^{\mathrm{A}}$ & $1.88 \times 10^{9 \mathrm{~A}}$ \\
\hline $4 f^{13} 5 d\left({ }^{3} D^{\circ}\right) 6 s^{4} D_{7 / 2}^{\circ}$ & $4 f^{13} 5 d\left({ }^{3} F^{\circ}\right) 6 p^{4} F_{9 / 2}$ & $2193.49^{\mathrm{A}}$ & $0.269^{\mathrm{A}}$ & $3.72 \times 10^{8 \mathrm{~A}}$ \\
\hline $4 f^{13} 5 d^{2}\left({ }^{3} F^{\circ}\right){ }^{4} I_{13 / 2}^{\circ}$ & $4 f^{13} 5 d\left({ }^{3} H^{\circ}\right) 6 p{ }^{4} I_{13 / 2}$ & $2193.57^{\mathrm{A}}$ & $1.439^{\mathrm{A}}$ & $2.00 \times 10^{9 \mathrm{~A}}$ \\
\hline $4 f^{13} 5 d\left({ }^{3} D^{\circ}\right) 6 s^{4} D_{5 / 2}^{\circ}$ & $4 f^{13} 5 d\left({ }^{3} P^{\circ}\right) 6 p^{4} D_{5 / 2}$ & $2198.49^{\mathrm{A}}$ & $0.183^{\mathrm{A}}$ & $2.53 \times 10^{8 \mathrm{~A}}$ \\
\hline $4 f^{13} 5 d\left({ }^{3} D^{\circ}\right) 6 s^{4} D_{7 / 2}^{\circ}$ & $4 f^{13} 5 d\left({ }^{3} D^{\circ}\right) 6 p^{4} P_{5 / 2}$ & $2206.08^{\mathrm{A}}$ & $0.241^{\mathrm{A}}$ & $3.30 \times 10^{8 \mathrm{~A}}$ \\
\hline $4 f^{13} 5 d\left({ }^{3} P^{\circ}\right) 6 s{ }^{4} P_{3 / 2}^{\circ}$ & $4 f^{13} 5 d\left({ }^{3} P^{\circ}\right) 6 p{ }^{4} P_{3 / 2}$ & $2210.28^{\mathrm{A}}$ & $0.153^{\mathrm{A}}$ & $2.08 \times 10^{8 \mathrm{~A}}$ \\
\hline $4 f^{13} 5 d\left({ }^{3} P^{\circ}\right) 6 s{ }^{4} P_{3 / 2}^{\circ}$ & $4 f^{13} 5 d\left({ }^{3} F^{\circ}\right) 6 p^{2} D_{5 / 2}$ & $2211.34^{\mathrm{A}}$ & $0.181^{\mathrm{A}}$ & $2.47 \times 10^{8 \mathrm{~A}}$ \\
\hline $4 f^{13} 5 d\left({ }^{3} P^{\circ}\right) 6 s{ }^{4} P_{1 / 2}^{\circ}$ & $4 f^{13} 5 d\left({ }^{3} P^{\circ}\right) 6 p^{4} P_{1 / 2}$ & $2225.28^{\mathrm{A}}$ & $0.585^{\mathrm{A}}$ & $7.88 \times 10^{8 \mathrm{~A}}$ \\
\hline $4 f^{13} 5 d\left({ }^{3} P^{\circ}\right) 6 s^{2} P_{3 / 2}^{\circ}$ & $4 f^{13} 5 d\left({ }^{3} P^{\circ}\right) 6 p{ }^{4} P_{1 / 2}$ & $2227.37^{\mathrm{A}}$ & $0.722^{\mathrm{A}}$ & $9.71 \times 10^{8 \mathrm{~A}}$ \\
\hline $4 f^{13} 5 d^{2}\left({ }^{3} F^{\circ}\right)^{2} I_{11 / 2}^{\circ}$ & $4 f^{13} 5 d\left({ }^{1} H^{\circ}\right) 6 p^{2} G_{9 / 2}$ & $2236.25^{\mathrm{A}}$ & $0.097^{\mathrm{A}}$ & $1.30 \times 10^{8 \mathrm{~A}}$ \\
\hline $4 f^{13} 5 d^{2}\left({ }^{3} F^{\circ}\right){ }^{4} F_{9 / 2}^{\circ}$ & $4 f^{13} 5 d\left({ }^{1} H^{\circ}\right) 6 p^{2} G_{9 / 2}$ & $2241.63^{\mathrm{A}}$ & $0.351^{\mathrm{A}}$ & $4.66 \times 10^{8 \mathrm{~A}}$ \\
\hline $4 f^{13} 5 d\left({ }^{3} D^{\circ}\right) 6 s^{4} D_{7 / 2}^{\circ}$ & $4 f^{13} 5 d\left({ }^{3} F^{\circ}\right) 6 p^{4} G_{9 / 2}$ & $2243.74^{\mathrm{A}}$ & $2.211^{\mathrm{A}}$ & $2.93 \times 10^{9 \mathrm{~A}}$ \\
\hline $4 f^{13} 5 d\left({ }^{3} D^{\circ}\right) 6 s^{4} D_{7 / 2}^{\circ}$ & $4 f^{13} 5 d\left({ }^{3} D^{\circ}\right) 6 p^{4} D_{7 / 2}$ & $2251.35^{\mathrm{A}}$ & $0.336^{\mathrm{A}}$ & $4.42 \times 10^{8 \mathrm{~A}}$ \\
\hline $4 f^{13} 5 d\left({ }^{3} D^{\circ}\right) 6 s^{4} D_{5 / 2}^{\circ}$ & $4 f^{13} 5 d\left({ }^{1} F^{\circ}\right) 6 p^{2} G_{7 / 2}$ & $2262.69^{\mathrm{A}}$ & $0.233^{\mathrm{A}}$ & $3.03 \times 10^{8 \mathrm{~A}}$ \\
\hline $4 f^{13} 5 d\left({ }^{3} P^{\circ}\right) 6 s{ }^{4} P_{3 / 2}^{\circ}$ & $4 f^{13} 5 d\left({ }^{3} P^{\circ}\right) 6 p^{2} S_{1 / 2}$ & $2263.01^{\mathrm{A}}$ & $0.091^{\mathrm{A}}$ & $1.18 \times 10^{8 \mathrm{~A}}$ \\
\hline $4 f^{13} 5 d\left({ }^{3} D^{\circ}\right) 6 s^{4} D_{5 / 2}^{\circ}$ & $4 f^{13} 5 d\left({ }^{3} D^{\circ}\right) 6 p{ }^{4} P_{5 / 2}$ & $2267.05^{\mathrm{A}}$ & $0.410^{\mathrm{A}}$ & $5.33 \times 10^{8 \mathrm{~A}}$ \\
\hline $4 f^{13} 5 d\left({ }^{3} P^{\circ}\right) 6 s^{2} P_{3 / 2}^{\circ}$ & $4 f^{13} 5 d\left({ }^{3} P^{\circ}\right) 6 p{ }^{4} S_{3 / 2}$ & $2267.31^{\mathrm{A}}$ & $0.949^{\mathrm{A}}$ & $1.23 \times 10^{9 \mathrm{~A}}$ \\
\hline $4 f^{13} 5 d\left({ }^{3} P^{\circ}\right) 6 s^{2} P_{3 / 2}^{\circ}$ & $4 f^{13} 5 d\left({ }^{3} P^{\circ}\right) 6 p{ }^{4} P_{5 / 2}$ & $2270.17^{\mathrm{A}}$ & $0.528^{\mathrm{A}}$ & $6.83 \times 10^{8 \mathrm{~A}}$ \\
\hline $4 f^{13} 5 d\left({ }^{3} P^{\circ}\right) 6 s{ }^{4} P_{1 / 2}^{\circ}$ & $4 f^{13} 5 d\left({ }^{3} P^{\circ}\right) 6 p{ }^{4} P_{3 / 2}$ & $2283.94^{\mathrm{A}}$ & $0.819^{\mathrm{A}}$ & $1.05 \times 10^{9 \mathrm{~A}}$ \\
\hline $4 f^{13} 5 d^{2}\left({ }^{3} F^{\circ}\right)^{2} I_{11 / 2}^{\circ}$ & $4 f^{13} 5 d\left({ }^{3} H^{\circ}\right) 6 p^{2} I_{11 / 2}$ & $2312.39^{\mathrm{A}}$ & $1.566^{\mathrm{A}}$ & $1.95 \times 10^{9 \mathrm{~A}}$ \\
\hline $4 f^{13} 5 d^{2}\left({ }^{3} F^{\circ}\right){ }^{4} F_{9 / 2}^{\circ}$ & $4 f^{13} 5 d\left({ }^{1} H^{\circ}\right) 6 p^{2} I_{11 / 2}$ & $2318.15^{\mathrm{A}}$ & $0.130^{\mathrm{A}}$ & $1.61 \times 10^{8 \mathrm{~A}}$ \\
\hline $4 f^{13} 5 d\left({ }^{3} D^{\circ}\right) 6 s^{4} D_{7 / 2}^{\circ}$ & $4 f^{13} 5 d\left({ }^{1} H^{\circ}\right) 6 p^{2} H_{9 / 2}$ & $2318.76^{\mathrm{A}}$ & $0.119^{\mathrm{A}}$ & $1.48 \times 10^{8 \mathrm{~A}}$ \\
\hline $4 f^{13} 5 d^{2}\left({ }^{3} F^{\circ}\right){ }^{4} F_{7 / 2}^{\circ}$ & $4 f^{13} 5 d\left({ }^{3} P^{\circ}\right) 6 p^{2} D_{5 / 2}$ & $2319.33^{\mathrm{A}}$ & $0.947^{\mathrm{A}}$ & $1.17 \times 10^{9 \mathrm{~A}}$ \\
\hline \multirow[t]{2}{*}{$4 f^{14} 5 f^{2} F_{5 / 2}^{\circ}$} & $4 f^{14} 7 g^{2} G_{7 / 2}$ & $2329.59^{\mathrm{A}}$ & $0.361^{\mathrm{A}}$ & $4.44 \times 10^{8 \mathrm{~A}}$ \\
\hline & & $2332.97^{\mathrm{B}}$ & $0.393^{\mathrm{B}}$ & $4.82 \times 10^{8 \mathrm{~B}}$ \\
\hline \multirow[t]{2}{*}{$4 f^{14} 5 f^{2} F_{7 / 2}^{\circ}$} & $4 f^{14} 7 g^{2} G_{7 / 2}$ & $2348.82^{\mathrm{A}}$ & $0.013^{\mathrm{A}}$ & $1.53 \times 10^{7 \mathrm{~A}}$ \\
\hline & & $2339.16^{\mathrm{B}}$ & $0.015^{\mathrm{B}}$ & $1.77 \times 10^{7 \mathrm{~B}}$ \\
\hline \multirow[t]{2}{*}{$4 f^{14} 5 f^{2} F_{7 / 2}^{\circ}$} & $4 f^{14} 7 g^{2} G_{9 / 2}$ & $2341.17^{\mathrm{A}}$ & $0.523^{\mathrm{A}}$ & $6.36 \times 10^{8 \mathrm{~A}}$ \\
\hline & & $2339.14^{\mathrm{B}}$ & $0.508^{\mathrm{B}}$ & $6.20 \times 10^{8 \mathrm{~B}}$ \\
\hline $4 f^{13} 5 d\left({ }^{3} P^{\circ}\right) 6 s{ }^{4} P_{3 / 2}^{\circ}$ & $4 f^{13} 5 d\left({ }^{1} D^{\circ}\right) 6 p^{2} F_{5 / 2}$ & $2372.04^{\mathrm{A}}$ & $0.151^{\mathrm{A}}$ & $1.79 \times 10^{8 \mathrm{~A}}$ \\
\hline $4 f^{13} 5 d\left({ }^{3} D^{\circ}\right) 6 s^{4} D_{5 / 2}^{\circ}$ & $4 f^{13} 5 d\left({ }^{3} D^{\circ}\right) 6 p{ }^{4} P_{3 / 2}$ & $2374.81^{\mathrm{A}}$ & $1.181^{\mathrm{A}}$ & $1.40 \times 10^{9 \mathrm{~A}}$ \\
\hline $4 f^{13} 5 d\left({ }^{3} D^{\circ}\right) 6 s^{4} D_{5 / 2}^{\circ}$ & $4 f^{13} 5 d\left({ }^{1} D^{\circ}\right) 6 p^{2} F_{5 / 2}$ & $2397.44^{\mathrm{A}}$ & $0.411^{\mathrm{A}}$ & $4.76 \times 10^{8 \mathrm{~A}}$ \\
\hline $4 f^{13} 5 d^{2}\left({ }^{3} F^{\circ}\right){ }^{4} P_{3 / 2}^{\circ}$ & $4 f^{13} 5 d\left({ }^{3} P^{\circ}\right) 6 p{ }^{4} P_{3 / 2}$ & $2404.06^{\mathrm{A}}$ & $0.268^{\mathrm{A}}$ & $3.09 \times 10^{8 \mathrm{~A}}$ \\
\hline $4 f^{13} 5 d\left({ }^{3} P^{\circ}\right) 6 s{ }^{4} P_{3 / 2}^{\circ}$ & $4 f^{13} 5 d\left({ }^{3} P^{\circ}\right) 6 p^{4} D_{3 / 2}$ & $2415.52^{\mathrm{A}}$ & $0.901^{\mathrm{A}}$ & $1.03 \times 10^{9 \mathrm{~A}}$ \\
\hline \multirow[t]{2}{*}{$4 f^{14} 5 f^{2} F_{5 / 2}^{\circ}$} & $4 f^{14} 9 d^{2} D_{5 / 2}$ & $2437.21^{\mathrm{A}}$ & $0.001^{\mathrm{A}}$ & $1.65 \times 10^{6 \mathrm{~A}}$ \\
\hline & & $2442.01^{\mathrm{B}}$ & $0.001^{\mathrm{B}}$ & $1.37 \times 10^{6 \mathrm{~B}}$ \\
\hline \multirow[t]{2}{*}{$4 f^{14} 5 f^{2} F_{5 / 2}^{\circ}$} & $4 f^{14} 9 d^{2} D_{3 / 2}$ & $2441.02^{\mathrm{A}}$ & $0.017^{\mathrm{A}}$ & $1.92 \times 10^{7 \mathrm{~A}}$ \\
\hline & & $2448.46^{\mathrm{B}}$ & $0.017^{\mathrm{B}}$ & $1.90 \times 10^{7 \mathrm{~B}}$ \\
\hline \multirow{3}{*}{$\begin{array}{l}4 f^{13} 5 d\left({ }^{3} D^{\circ}\right) 6 s^{4} D_{5 / 2}^{\circ} \\
4 f^{14} 5 f^{2} F_{7 / 2}^{\circ}\end{array}$} & $4 f^{13} 5 d\left({ }^{3} P^{\circ}\right) 6 p^{4} D_{3 / 2}$ & $2441.87^{\mathrm{A}}$ & $0.108^{\mathrm{A}}$ & $1.21 \times 10^{8 \mathrm{~A}}$ \\
\hline & $4 f^{14} 9 d^{2} D_{5 / 2}$ & $2451.47^{\mathrm{A}}$ & $0.043^{\mathrm{A}}$ & $4.76 \times 10^{7 \mathrm{~A}}$ \\
\hline & & $2448.80^{\mathrm{B}}$ & $0.024^{\mathrm{B}}$ & $2.71 \times 10^{7 \mathrm{~B}}$ \\
\hline
\end{tabular}


TABLE II (cont.)

\begin{tabular}{|c|c|c|c|c|}
\hline \multicolumn{2}{|c|}{ Transition } & $\lambda[\AA]$ & $g f$ & $g A_{k i}\left[\mathrm{~s}^{-1}\right]$ \\
\hline Lower level & Upper level & \multicolumn{3}{|c|}{ This work } \\
\hline $4 f^{13} 5 d\left({ }^{3} P^{\circ}\right) 6 s^{4} P_{5 / 2}^{\circ}$ & $4 f^{13} 5 d\left({ }^{3} P^{\circ}\right) 6 p^{2} D_{5 / 2}$ & $2471.38^{\mathrm{A}}$ & $1.154^{\mathrm{A}}$ & $1.26 \times 10^{9 \mathrm{~A}}$ \\
\hline $4 f^{13} 5 d\left({ }^{3} D^{\circ}\right) 6 s^{4} D_{5 / 2}^{\circ}$ & $4 f^{13} 5 d\left({ }^{3} F^{\circ}\right) 6 p^{4} D_{7 / 2}$ & $2493.91^{\mathrm{A}}$ & $0.247^{\mathrm{A}}$ & $2.65 \times 10^{8 \mathrm{~A}}$ \\
\hline $4 f^{13} 5 d\left({ }^{3} P^{\circ}\right) 6 s{ }^{4} P_{1 / 2}^{\circ}$ & $4 f^{13} 5 d\left({ }^{3} P^{\circ}\right) 6 p^{4} D_{3 / 2}$ & $2503.77^{\mathrm{A}}$ & $0.351^{\mathrm{A}}$ & $3.74 \times 10^{8 \mathrm{~A}}$ \\
\hline $4 f^{13} 5 d\left({ }^{3} P^{\circ}\right) 6 s{ }^{4} P_{3 / 2}^{\circ}$ & $4 f^{13} 5 d\left({ }^{3} P^{\circ}\right) 6 p^{4} P_{1 / 2}$ & $2554.76^{\mathrm{A}}$ & $0.285^{\mathrm{A}}$ & $2.91 \times 10^{8 \mathrm{~A}}$ \\
\hline $4 f^{13} 5 d\left({ }^{3} P^{\circ}\right) 6 s{ }^{4} P_{5 / 2}^{\circ}$ & $4 f^{13} 5 d\left({ }^{3} P^{\circ}\right) 6 p^{4} P_{3 / 2}$ & $2557.08^{\mathrm{A}}$ & $1.385^{\mathrm{A}}$ & $1.41 \times 10^{9 \mathrm{~A}}$ \\
\hline $4 f^{13} 5 d\left({ }^{3} D^{\circ}\right) 6 s{ }^{4} D_{7 / 2}^{\circ}$ & $4 f^{13} 5 d\left({ }^{3} P^{\circ}\right) 6 p^{4} P_{5 / 2}$ & $2559.59^{\mathrm{A}}$ & $0.816^{\mathrm{A}}$ & $8.31 \times 10^{8 \mathrm{~A}}$ \\
\hline $4 f^{13} 5 d\left({ }^{3} D^{\circ}\right) 6 s{ }^{4} D_{7 / 2}^{\circ}$ & $4 f^{13} 5 d\left({ }^{3} D^{\circ}\right) 6 p^{2} F_{7 / 2}$ & $2579.46^{\mathrm{A}}$ & $0.777^{\mathrm{A}}$ & $7.79 \times 10^{8 \mathrm{~A}}$ \\
\hline $4 f^{13} 5 d\left({ }^{3} P^{\circ}\right) 6 s^{2} P_{3 / 2}^{\circ}$ & $4 f^{13} 5 d\left({ }^{3} P^{\circ}\right) 6 p^{2} D_{5 / 2}$ & $2581.93^{\mathrm{A}}$ & $0.731^{\mathrm{A}}$ & $7.31 \times 10^{8 \mathrm{~A}}$ \\
\hline $4 f^{13} 5 d\left({ }^{3} D^{\circ}\right) 6 s{ }^{4} D_{5 / 2}^{\circ}$ & $4 f^{13} 5 d\left({ }^{3} P^{\circ}\right) 6 p^{4} P_{5 / 2}$ & $2612.96^{\mathrm{A}}$ & $0.114^{\mathrm{A}}$ & $1.11 \times 10^{8 \mathrm{~A}}$ \\
\hline $4 f^{13} 5 d\left({ }^{3} D^{\circ}\right) 6 s{ }^{4} D_{5 / 2}^{\circ}$ & $4 f^{13} 5 d\left({ }^{3} P^{\circ}\right) 6 p^{4} S_{3 / 2}$ & $2638.16^{\mathrm{A}}$ & $0.106^{\mathrm{A}}$ & $1.00 \times 10^{8 \mathrm{~A}}$ \\
\hline $4 f^{13} 5 d\left({ }^{3} D^{\circ}\right) 6 s{ }^{4} D_{5 / 2}^{\circ}$ & $4 f^{13} 5 d\left({ }^{3} D^{\circ}\right) 6 p^{2} F_{7 / 2}$ & $2663.20^{\mathrm{A}}$ & $0.203^{\mathrm{A}}$ & $1.91 \times 10^{8 \mathrm{~A}}$ \\
\hline \multirow[t]{2}{*}{$4 f^{14} 5 f^{2} F_{5 / 2}^{\circ}$} & $4 f^{14} 6 g^{2} G_{7 / 2}$ & $2809.47^{\mathrm{A}}$ & $1.105^{\mathrm{A}}$ & $9.34 \times 10^{8 \mathrm{~A}}$ \\
\hline & & $2814.43^{\mathrm{B}}$ & $1.176^{\mathrm{B}}$ & $9.90 \times 10^{8 \mathrm{~B}}$ \\
\hline \multirow{2}{*}{$4 f^{14} 5 f^{2} F_{7 / 2}^{\circ}$} & $4 f^{14} 6 g^{2} G_{7 / 2}$ & $2828.43^{\mathrm{A}}$ & $0.039^{\mathrm{A}}$ & $3.29 \times 10^{7 \mathrm{~A}}$ \\
\hline & & $2823.44^{\mathrm{B}}$ & $0.043^{\mathrm{B}}$ & $3.63 \times 10^{7 \mathrm{~B}}$ \\
\hline \multirow{2}{*}{$4 f^{14} 5 f^{2} F_{7 / 2}^{\circ}$} & $4 f^{14} 6 g^{2} G_{9 / 2}$ & $2828.38^{\mathrm{A}}$ & $1.380^{\mathrm{A}}$ & $1.15 \times 10^{9 \mathrm{~A}}$ \\
\hline & & $2823.37^{\mathrm{B}}$ & $1.520^{\mathrm{B}}$ & $1.27 \times 10^{9 \mathrm{~B}}$ \\
\hline \multirow[t]{2}{*}{$4 f^{14} 6 d^{2} D_{3 / 2}$} & $4 f^{14} 8 p^{2} P_{3 / 2}^{\circ}$ & $2902.34^{\mathrm{A}}$ & $0.003^{\mathrm{A}}$ & $2.42 \times 10^{6 \mathrm{~A}}$ \\
\hline & & $2912.77^{\mathrm{B}}$ & $0.002^{\mathrm{B}}$ & $1.78 \times 10^{6 \mathrm{~B}}$ \\
\hline \multirow[t]{2}{*}{$4 f^{14} 6 d^{2} D_{5 / 2}$} & $4 f^{14} 8 p^{2} P_{3 / 2}^{\circ}$ & $2970.10^{\mathrm{A}}$ & $0.026^{\mathrm{A}}$ & $2.00 \times 10^{7 \mathrm{~A}}$ \\
\hline & & $2981.02^{\mathrm{B}}$ & $0.020^{\mathrm{B}}$ & $1.50 \times 10^{7 \mathrm{~B}}$ \\
\hline \multirow[t]{2}{*}{$4 f^{14} 6 d^{2} D_{3 / 2}$} & $4 f^{14} 8 p^{2} P_{1 / 2}^{\circ}$ & $2988.55^{\mathrm{A}}$ & $0.015^{\mathrm{A}}$ & $1.09 \times 10^{7 \mathrm{~A}}$ \\
\hline & & $3000.04^{\mathrm{B}}$ & $0.011^{\mathrm{B}}$ & $8.15 \times 10^{6 \mathrm{~B}}$ \\
\hline \multirow[t]{2}{*}{$4 f^{14} 5 f^{2} F_{5 / 2}^{\circ}$} & $4 f^{14} 8 d^{2} D_{5 / 2}$ & $3030.57^{\mathrm{A}}$ & $0.001^{\mathrm{A}}$ & $5.60 \times 10^{5 \mathrm{~A}}$ \\
\hline & & $3090.12^{\mathrm{B}}$ & $0.004^{\mathrm{B}}$ & $2.68 \times 10^{6 \mathrm{~B}}$ \\
\hline \multirow{2}{*}{$4 f^{14} 5 f^{2} F_{7 / 2}^{\circ}$} & $4 f^{14} 8 d^{2} D_{5 / 2}$ & $3052.64^{\mathrm{A}}$ & $0.028^{\mathrm{A}}$ & $2.04 \times 10^{7 \mathrm{~A}}$ \\
\hline & & $3100.99^{\mathrm{B}}$ & $0.076^{\mathrm{B}}$ & $5.30 \times 10^{7 \mathrm{~B}}$ \\
\hline \multirow[t]{2}{*}{$4 f^{14} 5 f^{2} F_{5 / 2}^{\circ}$} & $4 f^{14} 8 d^{2} D_{3 / 2}$ & $3058.17^{\mathrm{A}}$ & $0.019^{\mathrm{A}}$ & $1.33 \times 10^{7 \mathrm{~A}}$ \\
\hline & & $3107.19^{\mathrm{B}}$ & $0.053^{\mathrm{B}}$ & $3.69 \times 10^{7 \mathrm{~B}}$ \\
\hline \multirow[t]{2}{*}{$4 f^{14} 8 p^{2} P_{1 / 2}^{\circ}$} & $4 f^{14} 10 d^{2} D_{3 / 2}$ & $3910.16^{\mathrm{A}}$ & $0.126^{\mathrm{A}}$ & $5.48 \times 10^{7 \mathrm{~A}}$ \\
\hline & & $3810.84^{\mathrm{B}}$ & $0.138^{\mathrm{B}}$ & $6.32 \times 10^{7 \mathrm{~B}}$ \\
\hline \multirow[t]{2}{*}{$4 f^{14} 8 p^{2} P_{3 / 2}^{\circ}$} & $4 f^{14} 10 d^{2} D_{3 / 2}$ & $4068.26^{\mathrm{A}}$ & $0.024^{\mathrm{A}}$ & $9.72 \times 10^{6 \mathrm{~A}}$ \\
\hline & & $3961.62^{\mathrm{B}}$ & $0.026^{\mathrm{B}}$ & $11.25 \times 10^{6 \mathrm{~B}}$ \\
\hline \multirow{2}{*}{$4 f^{14} 8 p^{2} P_{3 / 2}^{\circ}$} & $4 f^{14} 10 d^{2} D_{5 / 2}$ & $4061.97^{\mathrm{A}}$ & $0.233^{\mathrm{A}}$ & $9.41 \times 10^{7 \mathrm{~A}}$ \\
\hline & & $3950.59^{\mathrm{B}}$ & $0.239^{\mathrm{B}}$ & $10.21 \times 10^{7 \mathrm{~B}}$ \\
\hline \multirow[t]{2}{*}{$4 f^{14} 6 f^{2} F_{5 / 2}^{\circ}$} & $4 f^{14} 10 d^{2} D_{5 / 2}$ & $4450.04^{\mathrm{A}}$ & $0.003^{\mathrm{A}, \mathrm{B}}$ & $1.02 \times 10^{6 \mathrm{~A}, \mathrm{~B}}$ \\
\hline & & $4364.39^{\mathrm{B}}$ & & \\
\hline \multirow[t]{2}{*}{$4 f^{14} 6 f^{2} F_{7 / 2}^{\circ}$} & $4 f^{14} 10 d^{2} D_{5 / 2}$ & $4451.28^{\mathrm{A}}$ & $0.060^{\mathrm{A}}$ & $2.03 \times 10^{7 \mathrm{~A}}$ \\
\hline & & $4316.65^{\mathrm{B}}$ & $0.059^{\mathrm{B}}$ & $2.10 \times 10^{7 \mathrm{~B}}$ \\
\hline \multirow[t]{2}{*}{$4 f^{14} 6 f^{2} F_{5 / 2}^{\circ}$} & $4 f^{14} 10 d^{2} D_{3 / 2}$ & $4457.58^{\mathrm{A}}$ & $0.044^{\mathrm{A}}$ & $1.48 \times 10^{7 \mathrm{~A}}$ \\
\hline & & $4377.86^{\mathrm{B}}$ & $0.041^{\mathrm{B}}$ & $1.41 \times 10^{7 \mathrm{~B}}$ \\
\hline \multirow[t]{2}{*}{$4 f^{14} 8 p^{2} P_{1 / 2}^{\circ}$} & $4 f^{14} 9 d^{2} D_{3 / 2}$ & $4828.04^{\mathrm{A}}$ & $0.410^{\mathrm{A}}$ & $1.17 \times 10^{8 \mathrm{~A}, \mathrm{~B}}$ \\
\hline & & $4812.72^{\mathrm{B}}$ & $0.405^{\mathrm{B}}$ & \\
\hline \multirow[t]{2}{*}{$4 f^{14} 8 p^{2} P_{1 / 2}^{\circ}$} & $4 f^{14} 10 s^{2} S_{1 / 2}$ & $5049.33^{\mathrm{A}}$ & $0.110^{\mathrm{A}}$ & $2.87 \times 10^{7 \mathrm{~A}}$ \\
\hline & & $5016.86^{\mathrm{B}}$ & $0.112^{\mathrm{B}}$ & $2.96 \times 10^{7 \mathrm{~B}}$ \\
\hline $4 f^{14} 8 p^{2} P_{3 / 2}^{\circ}$ & $4 f^{14} 9 d^{2} D_{5 / 2}$ & $5054.98^{\mathrm{A}}$ & $0.669^{\mathrm{A}}$ & $1.75 \times 10^{8 \mathrm{~A}}$ \\
\hline & & $5028.33^{\mathrm{B}}$ & $0.697^{\mathrm{B}}$ & $1.84 \times 10^{8 \mathrm{~B}}$ \\
\hline $4 f^{14} 8 p^{2} P_{3 / 2}^{\circ}$ & $4 f^{14} 9 d^{2} D_{3 / 2}$ & $5071.39^{\mathrm{A}}$ & $0.078^{\mathrm{A}}$ & $2.02 \times 10^{7 \mathrm{~A}}$ \\
\hline & & $5055.73^{\mathrm{B}}$ & $0.077^{\mathrm{B}}$ & $2.01 \times 10^{7 \mathrm{~B}}$ \\
\hline $4 f^{14} 6 f^{2} F_{5 / 2}^{\circ}$ & $4 f^{14} 7 g^{2} G_{7 / 2}$ & $5120.02^{\mathrm{A}}$ & $1.358^{\mathrm{A}}$ & $3.45 \times 10^{8 \mathrm{~A}}$ \\
\hline & & $5154.31^{\mathrm{B}}$ & $1.345^{\mathrm{B}}$ & $3.38 \times 10^{8 \mathrm{~B}}$ \\
\hline $4 f^{14} 6 f^{2} F_{7 / 2}^{\circ}$ & $4 f^{14} 7 g^{2} G_{9 / 2}$ & $5114.79^{\mathrm{A}}$ & $1.605^{\mathrm{A}}$ & $4.09 \times 10^{8 \mathrm{~A}}$ \\
\hline & & $5087.74^{\mathrm{B}}$ & $1.766^{\mathrm{B}}$ & $4.55 \times 10^{8 \mathrm{~B}}$ \\
\hline
\end{tabular}


TABLE II (cont.)

\begin{tabular}{|c|c|c|c|c|}
\hline \multicolumn{2}{|c|}{ Transition } & $\lambda[\AA]$ & $g f$ & $g A_{k i}\left[\mathrm{~s}^{-1}\right]$ \\
\hline Lower level & Upper & \multicolumn{3}{|c|}{ This work } \\
\hline $4 f^{14} 6 f^{2} F_{7 / 2}^{\circ}$ & $4 f^{14} 7 g^{2}$ & $\begin{array}{l}5121.66^{\mathrm{A}} \\
5087.85^{\mathrm{B}}\end{array}$ & $0.050^{\mathrm{A}, \mathrm{B}}$ & $\begin{array}{l}1.28 \times 10^{7 \mathrm{~A}} \\
1.30 \times 10^{7 \mathrm{~B}}\end{array}$ \\
\hline $4 f^{14} 5 g^{2} G_{7 / 2}$ & $4 f^{14} 8 f^{2} F_{7 / 2}^{\circ}$ & $\begin{array}{l}5148.13^{\mathrm{A}} \\
5148.11^{\mathrm{B}}\end{array}$ & $0.000^{\mathrm{A}, \mathrm{B}}$ & $\begin{array}{l}1.17 \times 10^{5 \mathrm{~A}} \\
1.28 \times 10^{5 \mathrm{~B}}\end{array}$ \\
\hline $4 f^{14} 8 p^{2} P_{3 / 2}^{\circ}$ & $4 f^{14} 10 s^{2} S_{1 / 2}$ & $\begin{array}{l}5316 . \\
5281 .\end{array}$ & $\begin{array}{l}0.209^{\mathrm{A}} \\
0.212^{\mathrm{B}}\end{array}$ & $\begin{array}{l}4.93 \times 10^{7 \mathrm{~A}} \\
5.07 \times 10^{7 \mathrm{~B}}\end{array}$ \\
\hline $4 f^{14} 6 f^{2} F_{5 / 2}^{\circ}$ & $4 f^{14} 9 d^{2} D_{3 / 2}$ & $\begin{array}{l}5691.00^{\mathrm{A}} \\
5753.89^{\mathrm{B}}\end{array}$ & $\begin{array}{l}0.126^{\mathrm{A}} \\
0.121^{\mathrm{B}}\end{array}$ & $\begin{array}{l}2.60 \times 10^{7 \mathrm{~A}} \\
2.45 \times 10^{7 \mathrm{~B}}\end{array}$ \\
\hline $4 f^{14} 6 f^{2} F_{5 / 2}^{\circ}$ & $4 f^{14} 9 d^{2} D_{5 / 2}$ & $\begin{array}{l}5670.34^{\mathrm{A}} \\
5718.44^{\mathrm{B}}\end{array}$ & $0.009^{\mathrm{A}, \mathrm{B}}$ & $\begin{array}{l}1.89 \times 10^{6 \mathrm{~A}} \\
1.78 \times 10^{6 \mathrm{~B}}\end{array}$ \\
\hline $4 f^{14} 6 f^{2} F_{7 / 2}^{\circ}$ & $4 f^{14} 9 d^{2} D_{5 / 2}$ & & $\begin{array}{l}0.183^{\mathrm{A}} \\
0.177^{\mathrm{B}}\end{array}$ & $\begin{array}{l}3.79 \times 10^{7 \mathrm{~A}} \\
3.72 \times 10^{7 \mathrm{~B}}\end{array}$ \\
\hline $4 f^{14} 8 d^{2} D_{3 / 2}$ & $4 f^{14} 9 f^{2} F_{5 / 2}^{\circ}$ & & $\begin{array}{l}0.286^{\mathrm{A}} \\
0.199^{\mathrm{B}}\end{array}$ & $\begin{array}{l}4.36 \times 10^{7 \mathrm{~A}} \\
3.21 \times 10^{7 \mathrm{~B}}\end{array}$ \\
\hline $4 f^{14} 8 d^{2} D_{3 / 2}$ & $4 f^{14} 9 f^{2} F_{7 / 2}^{\circ}$ & & $\begin{array}{l}0.456^{\mathrm{A}} \\
0.281^{\mathrm{B}}\end{array}$ & $\begin{array}{l}6.69 \times 10^{7 \mathrm{~A}} \\
4.44 \times 10^{7 \mathrm{~B}}\end{array}$ \\
\hline $4 f^{14} 8 d^{2} D_{5 / 2}$ & $4 f^{14} 9 f^{2} F_{5 / 2}^{\circ}$ & & $\begin{array}{l}0.023^{\mathrm{A}} \\
0.014^{\mathrm{B}}\end{array}$ & $\begin{array}{l}3.35 \times 10^{6 \mathrm{~A}} \\
2.22 \times 10^{6 \mathrm{~B}}\end{array}$ \\
\hline $4 f^{14} 8 p^{2} P_{1 / 2}^{\circ}$ & $4 f^{14} 8 d^{2} D_{3 / 2}$ & $\begin{array}{l}8035.21^{\mathrm{A}} \\
8251.06^{\mathrm{B}}\end{array}$ & $\begin{array}{l}2.392^{\mathrm{A}} \\
3.187^{\mathrm{B}}\end{array}$ & $\begin{array}{l}2.47 \times 10^{8 \mathrm{~A}} \\
3.12 \times 10^{8 \mathrm{~B}}\end{array}$ \\
\hline $4 f^{14} 6 g^{2} G_{7 / 2}$ & $4 f^{14} 9 f^{2} F_{7 / 2}^{\circ}$ & $\begin{array}{l}817 \\
817\end{array}$ & $0.001^{\mathrm{A}, \mathrm{B}}$ & $\begin{array}{l}1.33 \times 10^{5 \mathrm{~A}} \\
1.42 \times 10^{5 \mathrm{~B}}\end{array}$ \\
\hline $4 f^{14} 6 g^{2} G_{9 / 2}$ & $4 f^{14} 9 f^{2} F_{7 / 2}^{\circ}$ & & $\begin{array}{l}0.046^{\mathrm{A}} \\
0.050^{\mathrm{B}}\end{array}$ & $\begin{array}{l}4.64 \times 10^{6 \mathrm{~A}} \\
4.98 \times 10^{6 \mathrm{~B}}\end{array}$ \\
\hline $4 f^{14} 6 g^{2} G_{7 / 2}$ & $4 f^{14} 9 f^{2} F_{5 / 2}^{\circ}$ & A & $\begin{array}{l}0.036^{\mathrm{A}} \\
0.038^{\mathrm{B}}\end{array}$ & $\begin{array}{l}3.58 \times 10^{6 \mathrm{~A}} \\
3.83 \times 10^{6 \mathrm{~B}}\end{array}$ \\
\hline $4 f^{14} 6 f^{2} F_{5 / 2}^{\circ}$ & $4 f^{14} 6 g^{2} G_{7 / 2}$ & & $\begin{array}{l}6.530^{\mathrm{A}} \\
6.222^{\mathrm{B}}\end{array}$ & $\begin{array}{l}6.48 \times 10^{8 \mathrm{~A}} \\
6.04 \times 10^{8 \mathrm{~B}}\end{array}$ \\
\hline $4 f^{14} 6 f^{2} F_{7 / 2}^{\circ}$ & $4 f^{14} 6 g^{2} G_{9 / 2}$ & & $\begin{array}{l}8.474^{\mathrm{A}} \\
8.235^{\mathrm{B}}\end{array}$ & $\begin{array}{l}8.40 \times 10^{8 \mathrm{~A}} \\
8.34 \times 10^{8 \mathrm{~B}}\end{array}$ \\
\hline $4 f^{14} 6 f^{2} F_{7 / 2}^{\circ}$ & $4 f^{14} 6 g^{2} G_{7 / 2}$ & & $\begin{array}{l}0.242^{\mathrm{A}} \\
0.235^{\mathrm{B}}\end{array}$ & $\begin{array}{l}2.40 \times 10^{7 \mathrm{~A}} \\
2.38 \times 10^{7 \mathrm{~B}}\end{array}$ \\
\hline $4 f^{14} 8 p^{2} P_{3 / 2}^{\circ}$ & $4 f^{14} 8 d^{2} D_{5 / 2}$ & & $\begin{array}{l}3.830^{\mathrm{A}} \\
5.348^{\mathrm{B}}\end{array}$ & $\begin{array}{l}3.53 \times 10^{8 \mathrm{~A}} \\
4.55 \times 10^{8 \mathrm{~B}}\end{array}$ \\
\hline $4 f^{14} 8 p^{2} P_{3 / 2}^{\circ}$ & $4 f^{14} 8 d^{2} D_{3 / 2}$ & & $\begin{array}{l}0.430^{\mathrm{A}} \\
0.585^{\mathrm{B}}\end{array}$ & $\begin{array}{l}3.76 \times 10^{7 \mathrm{~A}} \\
4.83 \times 10^{7 \mathrm{~B}}\end{array}$ \\
\hline $4 f^{14} 8 p^{2} P_{1 / 2}^{\circ}$ & $4 f^{14} 9 s^{2} S_{1 / 2}$ & & $\begin{array}{l}1.060^{\mathrm{A}} \\
1.107^{\mathrm{B}}\end{array}$ & $\begin{array}{l}7.69 \times 10^{7 \mathrm{~A}} \\
8.22 \times 10^{7 \mathrm{~B}}\end{array}$ \\
\hline $4 f^{14} 7 f^{2} F_{5 / 2}^{\circ}$ & $4 f^{14} 10 d^{2} D_{5 / 2}$ & $\begin{array}{l}968 \\
917\end{array}$ & $0.014^{\mathrm{A}, \mathrm{B}}$ & $\begin{array}{l}1.00 \times 10^{6 \mathrm{~A}} \\
1.15 \times 10^{6 \mathrm{~B}}\end{array}$ \\
\hline $4 f^{14} 7 f^{2} F_{7 / 2}^{\circ}$ & $4 f^{14} 10 d^{2} D_{5 / 2}$ & & $\begin{array}{l}0.281^{\mathrm{A}} \\
0.289^{\mathrm{B}}\end{array}$ & $\begin{array}{l}1.99 \times 10^{7 \mathrm{~A}} \\
2.28 \times 10^{7 \mathrm{~B}}\end{array}$ \\
\hline $4 f^{14} 7 f^{2} F_{5 / 2}^{\circ}$ & $4 f^{14} 10 d^{2} D_{3 / 2}$ & $\begin{array}{l}972 \\
923\end{array}$ & $\begin{array}{l}0.200^{\mathrm{A}} \\
0.201^{\mathrm{B}}\end{array}$ & $\begin{array}{l}1.41 \times 10^{7 \mathrm{~A}} \\
1.57 \times 10^{7 \mathrm{~B}}\end{array}$ \\
\hline $4 f^{14} 8 d^{2} D_{3 / 2}$ & $4 f^{14} 8 f^{2} F_{5 / 2}^{\circ}$ & $\begin{array}{l}9722.15^{\mathrm{A}} \\
9311.20^{\mathrm{B}}\end{array}$ & $\begin{array}{l}0.439^{\mathrm{A}} \\
0.348^{\mathrm{B}}\end{array}$ & $\begin{array}{l}3.10 \times 10^{7 \mathrm{~A}} \\
2.68 \times 10^{7 \mathrm{~B}}\end{array}$ \\
\hline $4 f^{14} 8 d^{2} D_{5 / 2}$ & $4 f^{14} 8 f^{2} F_{7 / 2}^{\circ}$ & $\begin{array}{l}9995.66^{\mathrm{A}} \\
9453.49^{\mathrm{B}}\end{array}$ & $\begin{array}{l}0.685^{\mathrm{A}} \\
0.490^{\mathrm{B}}\end{array}$ & $\begin{array}{l}4.58 \times 10^{7 \mathrm{~A}} \\
3.66 \times 10^{7 \mathrm{~B}}\end{array}$ \\
\hline $4 f^{14} 8 d^{2} D_{5 / 2}$ & $4 f^{14} 8 f^{2} F_{5 / 2}^{\circ}$ & $\begin{array}{l}10012.02^{\mathrm{A}} \\
9467.90^{\mathrm{B}}\end{array}$ & $\begin{array}{l}0.034^{\mathrm{A}} \\
0.024^{\mathrm{B}}\end{array}$ & $\begin{array}{l}2.29 \times 10^{6 \mathrm{~A}} \\
1.82 \times 10^{6 \mathrm{~B}}\end{array}$ \\
\hline $4 f^{14} 6 f^{2} F_{5 / 2}^{\circ}$ & $4 f^{14} 8 d^{2} D_{3 / 2}$ & $\begin{array}{l}10747.51^{\mathrm{A}} \\
11466.64^{\mathrm{B}}\end{array}$ & $\begin{array}{l}0.688^{\mathrm{A}} \\
0.970^{\mathrm{B}}\end{array}$ & $\begin{array}{l}3.97 \times 10^{7 \mathrm{~A}} \\
4.92 \times 10^{7 \mathrm{~B}}\end{array}$ \\
\hline $4 f^{14} 6 f$ & $4 f^{14} 8 d^{2} D_{5 / 2}$ & $\begin{array}{l}10414.20^{\mathrm{A}} \\
11237.60^{\mathrm{B}}\end{array}$ & $\begin{array}{l}0.046^{\mathrm{A}} \\
0.071^{\mathrm{B}}\end{array}$ & $\begin{array}{l}2.86 \times 10^{6 \mathrm{~A}} \\
3.74 \times 10^{6 \mathrm{~B}}\end{array}$ \\
\hline
\end{tabular}


TABLE II (cont.)

\begin{tabular}{|c|c|c|c|c|}
\hline \multicolumn{2}{|c|}{ Transition } & $\lambda[\AA]$ & $g f$ & $g A_{k i}\left[\mathrm{~s}^{-1}\right]$ \\
\hline Lower level & Upper level & \multicolumn{3}{|c|}{ This work } \\
\hline \multirow{2}{*}{$4 f^{14} 6 f^{2} F_{7 / 2}^{\circ}$} & $4 f^{14} 8 d^{2} D_{5 / 2}$ & $10421.00^{\mathrm{A}}$ & $0.928^{\mathrm{A}}$ & $5.70 \times 10^{7 \mathrm{~A}}$ \\
\hline & & $10926.46^{\mathrm{B}}$ & $1.455^{\mathrm{B}}$ & $8.13 \times 10^{7 \mathrm{~B}}$ \\
\hline \multirow{2}{*}{$4 f^{14} 8 p^{2} P_{3 / 2}^{\circ}$} & $4 f^{14} 9 s^{2} S_{1 / 2}$ & $10600.72^{\mathrm{A}}$ & $1.898^{\mathrm{A}}$ & $1.13 \times 10^{8 \mathrm{~A}}$ \\
\hline & & $10463.86^{\mathrm{B}}$ & $2.004^{\mathrm{B}}$ & $1.22 \times 10^{8 \mathrm{~B}}$ \\
\hline \multirow{2}{*}{$4 f^{14} 6 g^{2} G_{7 / 2}$} & $4 f^{14} 8 f^{2} F_{7 / 2}^{\circ}$ & $13499.73^{\mathrm{A}}$ & $0.008^{\mathrm{A}}$ & $2.87 \times 10^{5 \mathrm{~A}}$ \\
\hline & & $13498.74^{\mathrm{B}}$ & $0.009^{\mathrm{B}}$ & $3.15 \times 10^{5 \mathrm{~B}}$ \\
\hline \multirow{2}{*}{$4 f^{14} 6 g^{2} G_{9 / 2}$} & $4 f^{14} 8 f^{2} F_{7 / 2}^{\circ}$ & $13500.83^{\mathrm{A}}$ & $0.274^{\mathrm{A}}$ & $1.00 \times 10^{7 \mathrm{~A}}$ \\
\hline & & $13500.38^{\mathrm{B}}$ & $0.301^{\mathrm{B}}$ & $1.10 \times 10^{7 \mathrm{~B}}$ \\
\hline \multirow[t]{2}{*}{$4 f^{14} 6 g^{2} G_{7 / 2}$} & $4 f^{14} 8 f^{2} F_{5 / 2}^{\circ}$ & $13529.59^{\mathrm{A}}$ & $0.212^{\mathrm{A}}$ & $7.73 \times 10^{6 \mathrm{~A}}$ \\
\hline & & $13528.14^{\mathrm{B}}$ & $0.232^{\mathrm{B}}$ & $8.45 \times 10^{6 \mathrm{~B}}$ \\
\hline \multirow{2}{*}{$4 f^{14} 7 g^{2} G_{9 / 2}$} & $4 f^{14} 7 f^{2} F_{7 / 2}^{\circ}$ & $13534.31^{\mathrm{A}}$ & $6.975^{\mathrm{A}}$ & $2.54 \times 10^{8 \mathrm{~A}}$ \\
\hline & & $13582.06^{\mathrm{B}}$ & $7.494^{\mathrm{B}}$ & $2.71 \times 10^{8 \mathrm{~B}}$ \\
\hline \multirow[t]{2}{*}{$4 f^{14} 7 f^{2} F_{5 / 2}^{\circ}$} & $4 f^{14} 7 g^{2} G_{7 / 2}$ & $13540.82^{\mathrm{A}}$ & $6.032^{\mathrm{A}}$ & $2.19 \times 10^{8 \mathrm{~A}}$ \\
\hline & & $13541.05^{\mathrm{B}}$ & $5.798^{\mathrm{B}}$ & $2.11 \times 10^{8 \mathrm{~B}}$ \\
\hline \multirow[t]{2}{*}{$4 f^{14} 7 g^{2} G_{7 / 2}$} & $4 f^{14} 7 f^{2} F_{7 / 2}^{\circ}$ & $13582.50^{\mathrm{A}}$ & $0.223^{\mathrm{A}}$ & $8.07 \times 10^{6 \mathrm{~A}}$ \\
\hline & & $13582.90^{\mathrm{B}}$ & $0.214^{\mathrm{B}}$ & $7.74 \times 10^{6 \mathrm{~B}}$ \\
\hline \multirow[t]{2}{*}{$4 f^{14} 9 d^{2} D_{3 / 2}$} & $4 f^{14} 9 f^{2} F_{5 / 2}^{\circ}$ & $14601.47^{\mathrm{A}}$ & $0.317^{\mathrm{A}}$ & $9.90 \times 10^{6 \mathrm{~A}}$ \\
\hline & & $14470.00^{\mathrm{B}}$ & $0.279^{\mathrm{B}}$ & $8.88 \times 10^{6 \mathrm{~B}}$ \\
\hline \multirow[t]{2}{*}{$4 f^{14} 9 d^{2} D_{3 / 2}$} & $4 f^{14} 9 f^{2} F_{7 / 2}^{\circ}$ & $14715.84^{\mathrm{A}}$ & $0.365^{\mathrm{A}}$ & $1.13 \times 10^{7 \mathrm{~A}}$ \\
\hline & & $14675.77^{\mathrm{B}}$ & $0.393^{\mathrm{B}}$ & $1.22 \times 10^{7 \mathrm{~B}}$ \\
\hline \multirow[t]{2}{*}{$4 f^{14} 9 d^{2} D_{5 / 2}$} & $4 f^{14} 9 f^{2} F_{5 / 2}^{\circ}$ & $14739.26^{\mathrm{A}}$ & $0.018^{\mathrm{A}}$ & $5.62 \times 10^{5 \mathrm{~A}}$ \\
\hline & & $14699.18^{\mathrm{B}}$ & $0.020^{\mathrm{B}}$ & $6.05 \times 10^{5 \mathrm{~B}}$ \\
\hline \multirow[t]{2}{*}{$4 f^{14} 8 s^{2} S_{1 / 2}$} & $4 f^{14} 8 p^{2} P_{3 / 2}^{\circ}$ & $14302.46^{\mathrm{A}}$ & $2.670^{\mathrm{A}}$ & $8.71 \times 10^{7 \mathrm{~A}}$ \\
\hline & & $14559.43^{\mathrm{B}}$ & $2.690^{\mathrm{B}}$ & $8.46 \times 10^{7 \mathrm{~B}}$ \\
\hline
\end{tabular}

We obtained 11324 and 6937 possible electric dipole transitions between odd- and even-parity levels in the calculations $\mathrm{A}$ and $\mathrm{B}$, respectively. Table I shows the wavelengths, $\lambda$ (in $\AA$ ), the weighted oscillator strengths, $g f$, and the weighted transition rates (or probabilities), $g A_{k i}$ (in $\left.\mathrm{s}^{-1}\right)$, for $4 f^{14} n s(n=6-10)-4 f^{14} n p(n=6-10)$, $4 f^{14} n d(n=5-9)-4 f^{14} n p(n=6,7), 4 f^{14} n d(n=5-7)-$ $4 f^{14} n f(n=5-9)$, and $4 f^{14} 5 g-4 f^{14} n f(n=5,7-9)$ electric dipole (E1) transitions. The data obtained are too much. For this reason, we have here presented just a part of the results. The comparing values for these transitions exist in literature. Therefore, it is also made a comparison with other works in Table I.

In addition, we have reported the wavelengths, the weighted oscillator strengths and the weighted transition probabilities $g A_{k i}$ that are greater than or equal to $10^{5}$ for some new transitions $\left(4 f^{14} n p(n=6,7)-\right.$ $4 f^{14} 10 d, 4 f^{14} n d(n=5-10)-4 f^{14} 8 p, 4 f^{14} n s \quad(n=$ $8-10)-4 f^{14} 8 p, 4 f^{14} n g(n=5-7)-4 f^{14} n f(n=5-9)$, $\left.4 f^{14} n d(n=7-10)-4 f^{14} n f(n=5-9)\right)$ in Table II. We say again that a part of results of these transitions have been presented because there are too much results for $4 f^{13} 5 d^{2}-4 f^{13} 5 d 6 p, 4 f^{13} 5 d^{2}-4 f^{13} 6 s 6 p$,
$4 f^{13} 5 d 6 s-4 f^{13} 5 d 6 p$, and $4 f^{13} 5 d 6 s-4 f^{13} 6 s 6 p$ transitions $\left(g A_{k i} \geq 10^{8}\right)$ in Table II (all data can be obtained from corresponding author).

Our results presented in Table I are in excellent agreement with those of other works except some transitions. For some transitions, although the agreement is less in the weighted oscillator strengths and the weighted transition probabilities, it is very good in the wavelengths. The most of results obtained from this work are in agreement with literature $[1,6-8,11]$. In fact, we found for the values 1.109 (in calculation $\mathrm{A}$ ) and 1.129 (in calculation B) the mean ratio $g f$ (this work)/gf [11], except $4 f^{14} 6 s \quad{ }^{2} S_{1 / 2}-4 f^{14} 7 p \quad{ }^{2} P_{3 / 2}^{\circ}, \quad 4 f^{14} 5 d-4 f^{14} 7 p$, and $4 f^{14} 5 d-4 f^{14} 9 f$ the transitions. Again, except the $4 f^{14} 6 s{ }^{2} S_{1 / 2}-4 f^{14} 7 p^{2} P_{3 / 2}^{\circ}, 4 f^{14} 5 d-4 f^{14} 7 p$, $4 f^{14} 5 d-4 f^{14} 9 f$, and $4 f^{14} 6 p^{2} P_{3 / 2}^{\circ}-4 f^{14} 9 s{ }^{2} S_{1 / 2}$ transitions, we found also the values 1.094 (in calculation $\mathrm{A}$ ) and 1.143 (in calculation $\mathrm{B}$ ) for the mean ratio $g A_{k i}$ (this work) $/ g A_{k i}$ [11]. In addition, we calculated for the mean ratio $g A_{k i}$ (this work) $/ g A_{k i}$ [8, RMP calculation], the values 1.010 (in calculation $\mathrm{A}$ ) and 1.014 (in calculation B), except $4 f^{14} 6 p^{2} P_{3 / 2}^{\circ}-4 f^{14} 8 d, 4 f^{14} 6 p-4 f^{14} 9 d$ ${ }^{2} D_{3 / 2}, 4 f^{14} 6 s-4 f^{14} 7 p, 4 f^{14} 6 s-4 f^{14} n p(n=8-10)$, and 
$4 f^{14} 7 s-4 f^{14} 8 p^{2} P_{1 / 2}^{\circ}$ transitions. The transitions obtained from the calculation A agree with other works. This calculation includes core correlation. These results obtained from HFR calculations may be better, in case that the number configurations include the excitations from core. So the knowledge on the structure of these states is provided.

\section{Conclusion}

The main purpose of this paper was to perform HFR calculations for obtaining description of the Lu III spectrum. Accurate atomic structure data is an essential ingredient for a wide range of research fields. Areas from plasma research applications in nuclear fusion to lighting research, as well as astrophysics and cosmology, depend on such data. In spectrum synthesis works, particularly for CP stars, accurate data for transition probabilities (rates) and oscillator strengths for lanthanide atoms are needed to establish reliable abundances for these species. Our results for the radiative transitions for Lu III are in good agreement with other works. New results presented for the transitions between some excited levels are also reliable. There are a few experimental or theoretical radiative transition data for Lu III in literature. Consequently, we hope that our results, especially new results, obtained using the HFR method will be useful for research fields and technological applications, and other works in the future for Lu III spectra.

\section{Acknowledgments}

The authors are very grateful to the anonymous reviewer for stimulating comments and valuable suggestions, which resulted in improving the presentation of the paper.

\section{References}

[1] V. Kaufman, J. Sugar, J. Opt. Soc. Am. 61, 1693 (1971).

[2] W.F. Meggers, B.F. Scribner, J. Res. Nat. Bur. Stand. 5, 73 (1930).

[3] W.F. Meggers, B.F. Scribner, J. Res. Nat. Bur. Stand. A 19, 31 (1937).

[4] A. Steudel, Z. Phys. 152, 599 (1958).

[5] V. Kaufman, J. Sugar, J. Opt. Soc. Am. 66, 1019 (1976).

[6] J. Sugar, V. Kaufman, J. Opt. Soc. Am. 69, 141 (1979).
[7] J. Migdalek, J. Phys. B, At. Mol. Opt. Phys. 13, L169 (1980).

[8] J. Migdalek, J. Quant. Spectrosc. Radiat. Transfer 28, 417 (1982).

[9] K. Koc, J. Migdalek, J. Phys. B, At. Mol. Opt. Phys. 25, 907 (1992).

[10] P. Quinet, E. Biémont, At. Data Nucl. Data Tables 87, 207 (2004).

[11] E. Biémont, Z.S. Li, P. Palmeri, P. Quinet, J. Phys. B, At. Mol. Opt. Phys. 32, 3409 (1999).

[12] J.A. Fedchak, E.A. Den Hartog, J.E. Lawler, P. Palmeri, P. Quinet, E. Biémont, Astrophys. J. 542, 1109 (2000).

[13] www.tcd.ie/Physics/People/Cormac.McGuinness/ Cowan/ .

[14] R.D. Cowan, The Theory of Atomic Structure Spectra, University of California Press, USA 1981.

[15] B. Karaçoban, L. Özdemir, Chin. J. Phys., submitted for publication.

[16] B. Karaçoban, L. Özdemir, Acta Phys. Pol. A 113, 1609 (2008)

[17] B. Karaçoban, L. Özdemir, J. Quant. Spectrosc. Radiat. Transfer 109, 1968 (2008).

[18] B. Karaçoban, L. Özdemir, Acta Phys. Pol. A 115, 864 (2009).

[19] B. Karaçoban, L. Özdemir, Indian J. Phys. 84, 223 (2010).

[20] B. Karaçoban, L. Özdemir, Arab. J. Sci. Eng. 36, 635 (2011).

[21] B. Karaçoban, L. Özdemir, Cent. Eur. J. Phys. 9, 800 (2011).

[22] B. Karaçoban, L. Özdemir, Acta Phys. Pol. A 119, 342 (2011).

[23] B. Karaçoban, L. Özdemir, J. Korean Phys. Soc. 58, 417 (2011)

[24] B. Karaçoban, L. Özdemir, Z. Naturforsch. 66a, 543 (2011).

[25] B. Karaçoban, L. Özdemir, Indian J. Phys. 85, 683 (2011).

[26] B. Karaçoban, L. Özdemir, Cent. Eur. J. Phys. 10, 124 (2012).

[27] B. Karaçoban, L. Özdemir, Chin. J. Phys. 50, 49 (2012).

[28] C.F. Fischer, T. Brage, P. Jönsson, Computational Atomic Structure - An MCHF Approach, IOP, Bristol 1997.

[29] www.nist.gov/pml/data/asd.cfm . 